\title{
The Retromer Complex Protein VPS35 Modulates Iron Homeostasis by Regulating Hepcidin-Induced Ferroportin Degradation
}

\section{Permanent link}

http://nrs.harvard.edu/urn-3:HUL.InstRepos:37736757

\section{Terms of Use}

This article was downloaded from Harvard University's DASH repository, and is made available under the terms and conditions applicable to Other Posted Material, as set forth at http:// nrs.harvard.edu/urn-3:HUL.InstRepos:dash.current.terms-of-use\#LAA

\section{Share Your Story}

The Harvard community has made this article openly available.

Please share how this access benefits you. Submit a story.

Accessibility 
The Retromer Complex protein VPS35 Modulates Iron Homeostasis

by Regulating Hepcidin-induced Ferroportin Degradation

Zinger C. Yang

A Thesis in the Field of Biology

for the Degree of Master of Liberal Arts in Extension Studies

Harvard University

November 2017 



\begin{abstract}
Hepcidin-mediated degradation of the iron exporter Ferroportin is an essential mechanism for iron homeostasis. Upon Hepcidin binding to the Ferroportin receptor at the cell surface, Ferroportin is rapidly internalized and degraded. However, the underlying mechanism for the degradation has not been uncovered. Using a functional genomics approach, VPS35, a member of the Retromer Complex, is identified as an important regulator for Hepcidin-induced Ferroportin degradation. Cells with CRISPRinduced mutation in VPS35 demonstrated significantly reduced Ferroportin degradation in the presence of Hepcidin. Interestingly, VPS35 mutations and dysfunction of other members of the Retromer Complex have been associated with familial autosomaldominant Parkinson's disease, a progressive neurodegenerative disease with wellestablished iron accumulation phenotype. The result of this study reveals a previously unappreciated role for the Retromer Complex in the regulation of iron homeostasis.
\end{abstract}




\section{Acknowledgments}

I would like to thank my co-advisor Dr. Feng Cong from Novartis Institutes for Biomedical Research for recommending the research topic and provide the opportunity to visit his lab and gain hands-on experience on conducting a pooled CRISPR screen. My co-advisor Dr. Jessica Whited from Harvard Medical School provided invaluable discussion and critical review of the manuscript. Many thanks to Feng Cong lab members Bo Lu, Lei Ji, Hao Zeng and Olga Kheifets for laboratory technique guidance and assistance. I appreciate the high quality NGS results generated by Amy Janiak and Alicia Lindeman. I also thank Deborah Ahern-Ridlon, Yang Sullivan, Alan Ho and Akos Szilvasi at the FACS Core Facility, Novartis Institutes for Biomedical Research for FACS assistance. Dr. Joseph Loureiro has provided multiple manuscript discussions. Lastly, I am immensely grateful to many Novartis colleagues for their support throughout the Master's study. 


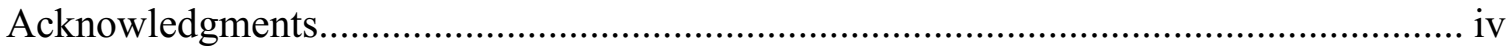

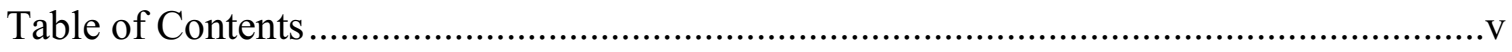

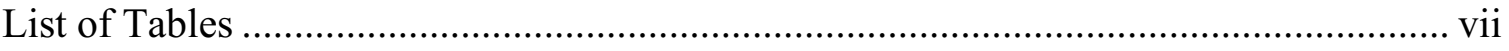

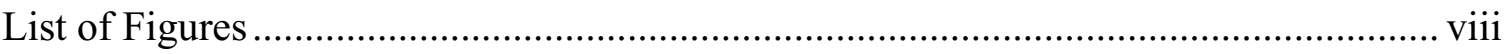

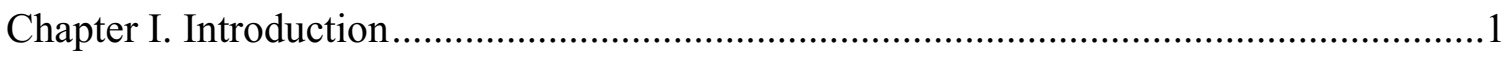

Chapter II. Materials and Methods …………………................................................5

Assay Development for the Ferroportin Pooled CRISPR Screen............................6

Generation of HEK293 Ferroportin Reporter Cell Line ...............................6

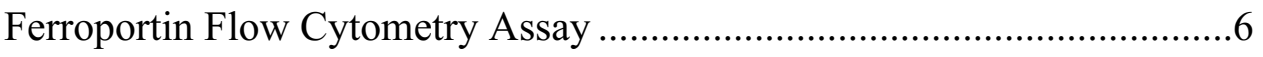

Cas9 Antibody Staining ......................................................................

Viral Titer Experiment ..........................................................................

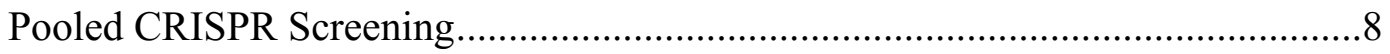

The Pooled CRISPR Library ...................................................................

The Pooled CRISPR Screen.....................................................................

NGS Library Construction and Sequencing ..............................................

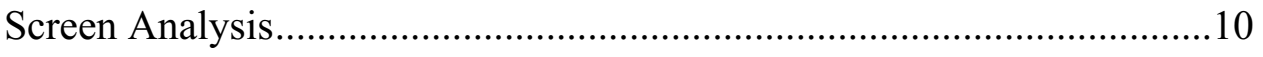

Validation Experiments ................................................................................ 10

CRISPR gRNA Lentiviral Packaging and Viral Infection ........................11

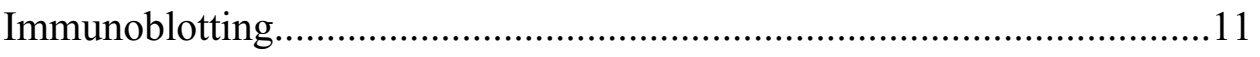

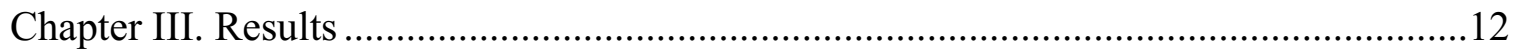

Developing the Ferroportin Pooled CRISPR Screening ........................................12 
Construction of the HEK293 Ferroportin Reporter Screening System .....12

Characterization of the FPN1-GFP Reporter System .............................13

Cas9 Characterization and Viral Titer ..............................................15

The Ferroportin Pooled CRISPR Screen .........................................................18

The Pooled CRISPR Screen............................................................... 18

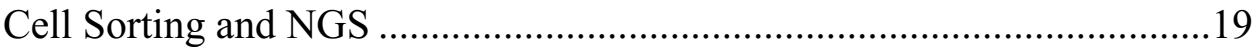

Statistical Analysis of the Screening Data ............................................20

Ferroportin Pooled CRISPR Screen Hit Selection and Validation .......................23

Transport Related Pathways Regulate Ferroportin-GFP level.................25

VPS35 is Important for Hepcidin-mediated Degradation of Ferroportin ..28

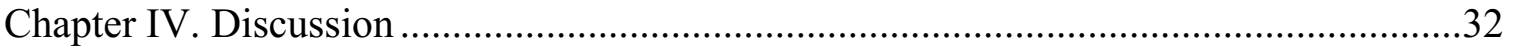

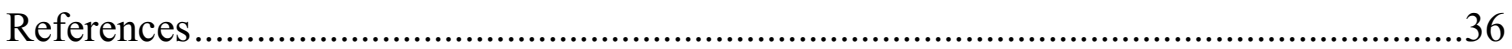




\section{List of Tables}

Table 1. VPS35 knockout cells have reduced sensitivity to Hepcidin - GFP quantitation. 


\section{List of Figures}

Figure 1. Ferroportin and Hepcidin regulates iron export....................................2

Figure 2. HEK293 Ferroportin (SLC40A1, FPN1)-GFP reporter construct.............13

Figure 3. Confocal microscopy image of FPN1-GFP in HEK293 _...........................14

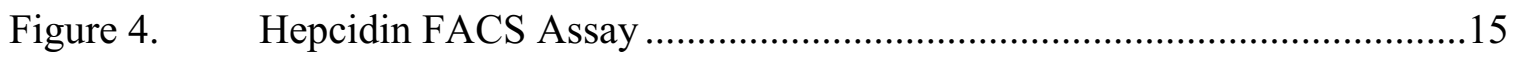

Figure 5. A single clone of HEK293::Tet-Off-FPN1-GFP::Cas9 cells have uniform

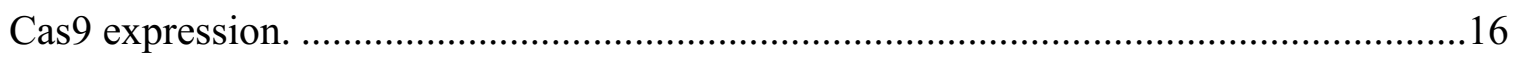

Figure 6. Determining the viral titer for HEK293::Tet-Off-FPN1-GFP::Cas9 -

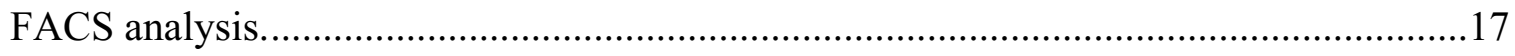

Figure 7. Determining the viral titer for HEK293::Tet-Off-FPN1-GFP::Cas9 - MOI

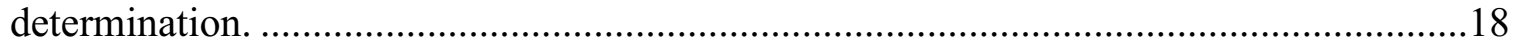

Figure 8. Schematic of Ferroportin-GFP pooled CRISPR screen..........................19

Figure 9. Post-sorting profile for subpopulations from the pooled CRISPR screen

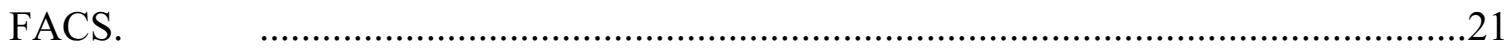

Figure 10. NGS and Quality Control for Pooled CRISPR Screen Results................22

Figure 11. The distribution of the differential gRNA representation.........................23

Figure 12. Ferroportin gRNAs were under-represented in the high FPN1-GFP sub-

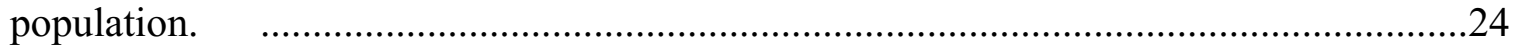

Figure 13. Positive regulators of Ferroportin identified from the pooled CRISPR

screen.

Figure 14. Negative regulators of Ferroportin identified from the pooled CRISPR

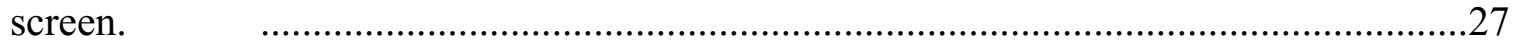

Figure 15. Pathways specifically enriched in the Hepcidin treated condition. .........28 
Figure 16. VPS35 knockout cells have reduced sensitivity to Hepcidin - FACS validation.

Figure 17. Western blot analysis of Ferroportin-GFP expression upon Hepcidin treatment. 


\section{Chapter I}

Introduction

Iron is an abundant element on earth and is involved in essential biological processes. With the ability to gain and lose electrons, iron participates in oxidative phosphorylation and other enzymatic processes. In the red blood cells of vertebrates, hemoglobin carries out its oxygen transport function by incorporating iron ions in its structure to bind to oxygen molecules. Deficiency of iron could lead to inadequate functional hemoglobin level in an organism and manifest as symptoms of anemia and fatigue. Yet iron in excess is toxic, and to prevent the body having dangerously low or dangerously high levels of iron in circulation, a tightly regulated iron homeostasis mechanism involving iron absorption, storage, and remobilization has evolved.

Ferroportin (FPN1; SLC40A1) is a cell surface transmembrane protein with 571 amino acids. The primary role of Ferroportin is to facilitate the transport of iron out of a cell into the circulation. In a mouse model where Ferroportin is inactivated, embryonic lethality is observed in extra-embryonic and embryonic tissues, presumably due to iron deficiency (Donovan, 2005). While the possibility remains that there exist other ion channels capable of iron export, none is sufficient to replace loss of Ferroportin (Donovan, 2005). Ferroportin is reported to be abundant in enterocytes, hepatocytes, and macrophages, although it is expressed in most tissues (Donovan, 2005). Nemeth, et al (2004) identified Hepcidin, a 25-amino-acid signaling peptide, as the key negative regulator of Ferroportin-mediated iron export. Hepcidin binds directly to Ferroportin at the cell surface and readily induces its internalization and subsequent degradation. This Ferroportin degradation process effectively blocks the cell from releasing iron into the 
circulation (Figure 1). Therefore, Hepcidin acts as an iron homeostasis regulatory protein through mediation of Ferroportin level.
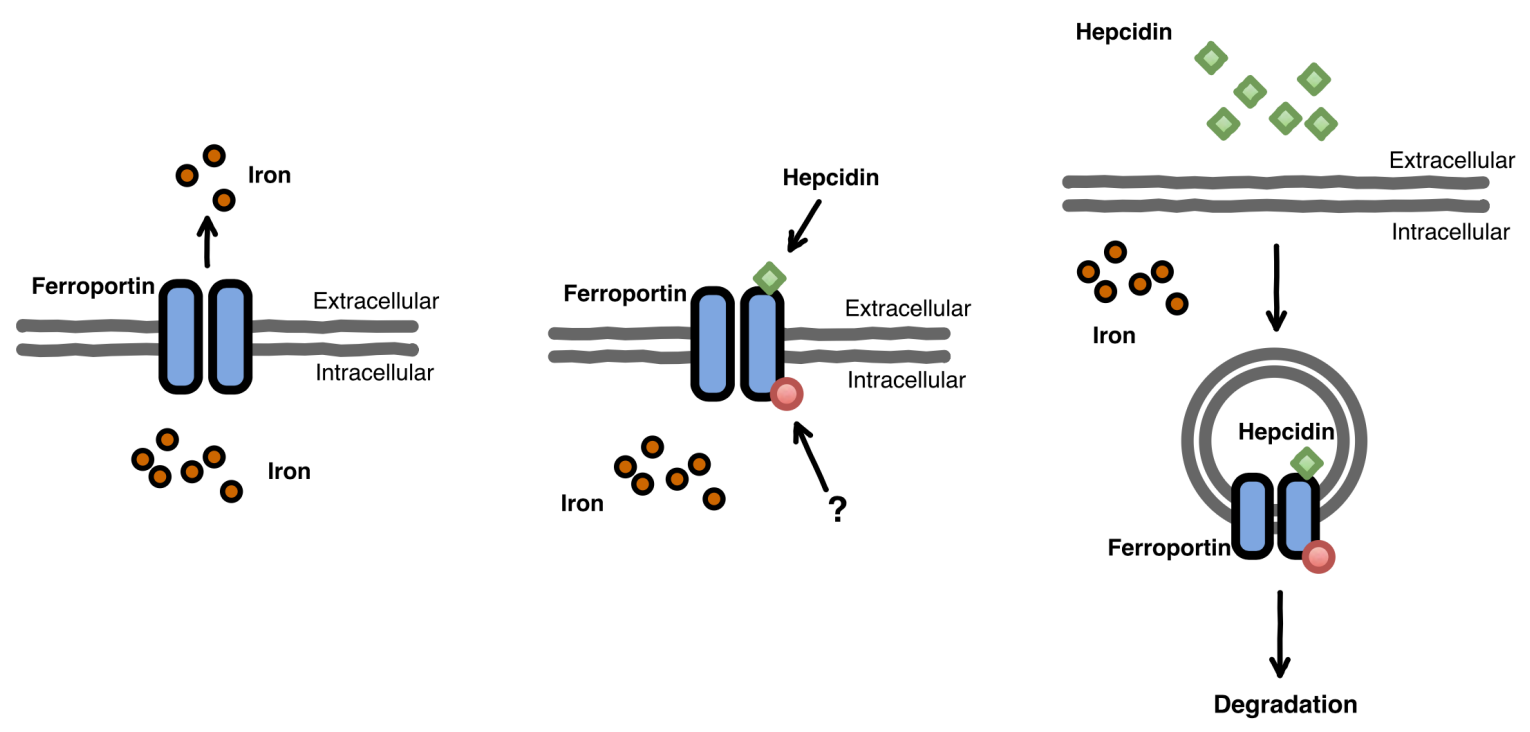

Figure 1. Ferroportin and Hepcidin regulates iron export.

Ferroportin is the sole exporter for cellular iron and its level at the cell surface is regulated by the iron regulatory hormone Hepcidin. Hepcidin binds directly to Ferroportin to induce its internalization and subsequent degradation while preventing iron from entering the circulation. Co-factors required for Ferroportin internalization and degradation have not yet been identified.

Human genetics studies have revealed the important role of Ferroportin in human health. Hemochromatosis type 4 (HFE4), also known as the Ferroportin disease, is caused by mutation in Ferroportin and is characterized by progressive iron overloading in which excessive iron accumulates in cells and leads to complications such as liver fibrosis and arrhythmia (Njajou, 2001; Montosi, 2001). HFE4 is an autosomal dominant disease, first identified in a large Italian pedigree (Pietrangelo, 1999). The patients are characterized by hepatic fibrosis, diabetes, impotence, and arrhythmias. Studies on HFE4 families revealed heterogeneity associated with different ferroportin mutations. Loss-of-function mutation 
in Ferroportin may result in a defect in its cell surface localization and loss of iron export function, revealed by clinical feature of anemia; gain-of-function mutation causes resistance to Hepcidin-induced degradation and leads to iron overload (Cemonesi, 2005; Sham, 2005; Drakesmith, 2005; Sham, 2009).

Iron could be internalized into cells via metal ion channel divalent metal transporter 1 (DMT1) and iron-recycling endocytosis, and the reactive metal are stored in Ferritin complexes which can retain up to 4000 iron ions per complex in containment and prevent iron-induced reactive oxidative stress. Additionally, a number of genes and pathways have been suggested to contribute to the overall systematic maintenance of iron homeostasis. How Ferroportin coordinates with the remainder of iron regulatory system to maintain circulatory iron homeostasis remains unknown. The present study focuses on identifying the molecular mechanism for Hepcidin-induced Ferroportin internalization and degradation.

In this study, an unbiased genetic screening approach was utilized to uncover novel mechanisms for Hepcidin-induced Ferroportin degradation. High-throughput whole genome pooled CRISPR screening is a powerful technology for functional genomics study (Wang, 2014). Pathway-based pooled CRISPR screening in a HEK293 cellular model with functional readout has demonstrated success in identifying new genes and pathway mechanisms (Wang, 2016). A stable cell line (HEK293::Tet-Off-FPN1GFP::Cas9) was established expressing human Ferroportin with a C-terminal green fluorescent protein (GFP) under the control of a Tet-Off inducible promoter. Cas9 was then stably expressed in HEK293::Tet-Off-FPN1-GFP cells to permit the CRISPR-based gene perturbation. Utilizing GFP level as the functional readout, CRISPR mutations 
differentially affecting FPN1-GFP level could be identified. Previous work on the roles of Hepcidin and Ferroportin in iron homeostasis have also been discovered on inducible FPN1-GFP cell lines, indicating that the HEK293 reporter system can serve as an appropriate platform for the study of Ferroportin.

The study aimed to identify regulators specific for the Hepcidin-induced degradation, thus the screen was designed comparing Hepcidin-treated to untreated conditions. Analysis of the results revealed VPS35 as a potent regulator of Hepcidinmediated Ferroportin degradation. VPS35, a core member of the Retromer Complex, was a screen hit specifically regulating FPN1-GFP level in the presence of Hepcidin. Validation results demonstrate that inhibition of VPS35 prevents Hepcidin-induced Ferroportin degradation. The Retromer Complex is reported to mediate transport and recycling of selected transmembrane receptors (Bonifacino, 2008). This study provides novel insights into Ferroportin degradation and suggests a potential role of the Retromer Complex in Ferroportin turnover and Hepcidin-mediated iron homeostasis. 


\section{Chapter II}

Materials and Methods

The aim of the study is to identify genes important for the internalization and degradation of Ferroportin using a pooled CRISPR screening approach. To develop the Ferroportin pooled CRISPR screen, a cell line with stable expression of Cas 9 and inducible FPN1-GFP reporter suitable for screening was established and characterized. A FACS assay was established to measure Hepcidin-induced degradation of FerroportinGFP.

The screening protocol resembled previously published phenotypic pooled CRISPR screen (Wang, 2016). The pooled CRISPR screening process began with culturing sufficient number of HEK293::Tet-Off-FPN1-GFP::Cas9 cells for infection with the pooled CRISPR library. On average 1000 cells per gRNA were transduced in the screening cells and the same coverage was maintained throughout the screen. Following sufficient days for CRISPR gene editing and cell growth, the screening cells were induced to express FPN1-GFP through doxycycline (Dox) withdrawal for two days, followed by 24 hours Hepcidin treatment. Cells were then subjected to fluorescenceactivated cell sorting (FACS) analysis to isolate high and low GFP sub-populations. Genomic DNA harvested from each sorted population was submitted to Next Generation Sequencing (NGS) for short-read deep sequencing of the CRISPR gRNA sequence. Custom scripts and published bioinformatics tools were utilized to process the raw NGS results and to identify the representation of library gRNAs in each of the harvested 
population. Statistical analysis revealed screen hits for follow-up and additional characterization.

Assay Development for the Ferroportin Pooled CRISPR Screen

Generation of HEK293 Ferroportin Reporter Cell Line

To generate HEK293::Tet-Off-FPN1-GFP, Human Ferroportin-GFP constructs were cloned into Retro- $\mathrm{X}^{\mathrm{TM}}$ Tet-Off® Advanced Inducible Expression System (Clontech, Mountain View, CA), and colonies were selected in the presence of $150 \mathrm{ug} / \mathrm{ml}$ hygromycin (Invitrogen, Carlsbad, CA) and $400 \mathrm{ug} / \mathrm{ml} \mathrm{G418} \mathrm{(Gibco,} \mathrm{Waltham,} \mathrm{MA).}$

To establish the Ferroportin reporter cell line for pooled CRISPR screening, HEK293::Tet-Off-FPN1-GFP cells were infected with lentivirus expressing Cas9, followed by selection with $20 \mathrm{ug} / \mathrm{ml}$ blasticidin (Sigma, St. Louis, MO). Cells were maintained in $10 \mathrm{ug} / \mathrm{ml}$ blasticidin.

Dox (Sigma, St. Louis, MO) was used to control the expression of FPN1-GFP. Hepcidin was purchased from Peptides International (Louisville, KY). HEK293 cells were grown in DMEM supplemented with 10\% FBS and penicillin/streptomycin (Invitrogen).

Ferroportin Flow Cytometry Assay

A FACS-based Ferroportin assay was established for the development of the CRISPR screen and for validation. In HEK293::Tet-Off-FPN1-GFP cells, FPN1-GFP expression was induced by Dox withdrawal for two days and followed by overnight Hepcidin treatment. HEK293::Tet-Off-FPN1-GFP with no FPN1-GFP induction served 
as the low GFP control. During harvest, cells were detached with TrypLE (Invitrogen). GFP level was quantified by CytoFLEX flow cytometer (Beckman Coulter Life Sciences). FlowJo software (FlowJo, LLC.) was used for FACS analysis.

\section{Cas9 Antibody Staining}

To confirm the expression of Cas9 and characterize Cas9 level in HEK293::TetOff-FPN1-GFP::Cas9, intracellular staining of Cas9 was performed along with the parental no-Cas9 cells. 3 million cells were fixed with $1 \%$ formaldehyde followed by fixation with ice-cold 100\% methanol. Cells were incubated in 1\% BSA, $0.2 \%$ Triton-PBS for 15 minutes to permeabilize the plasma membrane. The permeabilized cells were stained with Cas9 mouse monoclonal antibody with PE conjugate (Cell Signaling Technology 35193, Danvers, MA) and analyzed by FACS. CytoFLEX flow cytometer (Beckman Coulter Life Sciences) and FlowJo software (FlowJo, LLC.) were used for FACS analysis.

\section{Viral Titer Experiment}

To determine the multiplicity of infection (MOI) of gRNA lentiviral library, HEK293::TetOff-FPN1-GFP::Cas9 cells were plated in a 6 well plates at $1 \times 10^{5}$ cells per well. Twenty-four hours follow the plating, $1.6 \mathrm{uL}$ of polybrene and $0 \mathrm{ul}, 1 \mathrm{ul}, 3 \mathrm{ul}, 10 \mathrm{ul}$, $30 \mathrm{ul}, 100 \mathrm{ul}$ of reference virus carrying gRNA and RFP expression cassettes were added to individual wells. Four days after infection, RFP expression of each cell population was quantified by the CytoFLEX flow cytometer (Beckman Coulter Life Sciences). The amount of reference virus for MOI of 0.5 was interpolated using logarithmic curve fit. 


\section{Pooled CRISPR Screening}

The Pooled CRISPR Library

A genome-wide gRNA library with 92890 gRNAs targeting 19050 genes was adapted from published sequences (Wang, 2014), selecting the 5 gRNAs most proximal to transcription start site. gRNAs were synthesized using chip based oligonucleotide synthesis with fused tracRNA sequence (Chen, 2013) and cloned into pRSI16 lentiviral vector by Cellecta Inc. (Mountain View, CA).

\section{The Pooled CRISPR Screen}

Two hundred and twenty million HEK293 cells with constitutive Cas9 expression and Tet-Off Ferroportin-GFP reporter were transduced with the whole genome pooled CRISPR library at MOI $=0.5$. Cells were maintained at approximately $1000 \mathrm{x}$ coverage of the lentiviral library throughout the screen. Nine days after puromycin selection for viral transduction, Dox was withdrawn to induce Ferroportin-GFP expression. Eleven days after selection, cells were treated with $1 \mathrm{ug} / \mathrm{ml}$ Hepcidin to induce Ferroportin-GFP degradation. Dox was added at the same time as Hepcidin treatment to stop the transcription of Ferroportin-GFP. For the untreated group, only Dox was added. After 24 hours of treatment, 25\% high GFP and 25\% low GFP cells were collected using BD FACS Aria Cell Sorter. Genomic DNA was collected and subjected to Illumina DNA sequencing. 
NGS Library Construction and Sequencing

Genomic DNA from live cells was isolated using the QIAamp DNA Blood Maxi kit (Qiagen) and quantified using PicoGreen (Invitrogen). Illumina sequencing libraries were generated using PCR amplification with primers specific to the genome integrated lentiviral vector backbone sequence. A total of 24 independent $100 \mu \mathrm{L}$ PCR reactions were performed per transduced sample. Each PCR reaction contained $4 \mu \mathrm{g}$ of genomic DNA (a total of $96 \mu \mathrm{g}$ genomic DNA was used per transduced sample), and was performed with Titanium Taq (Clontech) using the following PCR primers (Integrated DNA Technologies):

5644(5’AATGATACGGCGACCACCGAGATCTACACTCGATTTCTTGGCTT TATA TATCTTGTGGAAAGGA-3’)

Index(5'CAAGCAGAAGACGGCATACGAGATXXXXXXXXXXGTGACTGG AGTT CAGACGTGTGCTCTTCCGA TC-3’)

X denotes a 10 base PCR-sample specific barcode used for data demultiplexing following sequencing. PCR cycling conditions were as follows: $1 \mathrm{x} 98^{\circ} \mathrm{C}$ for $5 \mathrm{~min}$; $28 \mathrm{x}$ $95^{\circ} \mathrm{C}$ for $15 \mathrm{sec}, 65^{\circ} \mathrm{C}$ for $15 \mathrm{sec}, 72^{\circ} \mathrm{C}$ for $30 \mathrm{sec}$; $1 \mathrm{x} 72^{\circ} \mathrm{C}$ for $5 \mathrm{~min}$. The resulting Illumina libraries were purified using 1.8x SPRI AMPure XL beads (Beckman Coulter) following the manufacturer's recommendations and qPCR quantified using primers specific to the Illumina sequences using standard methods. Illumina sequencing libraries were then pooled and sequenced with a HiSeq 2500 instrument (Illumina) using a custom read 1 sequencing primer $5645\left(5^{\prime}-\right.$

TCGATTTCTTGGCTTTATATATCTTGTGGAAAGGACGAAACACCG-3'), and a 11 base-pair index read, using a standard Illumina indexing primer ( $5^{\prime}-$ 
GATCGGAAGAGCACACGTCTGAACTCCAGTCAC-3'). Approximately 50 million reads were generated per transduced sample, resulting in an average of over 500 reads per gRNA.

Screen Analysis

Raw sequencing reads were converted to FASTQ format using the bc12fastq2 Conversion Software v2.17 (Illumina). The reads were trimmed and aligned to the gRNA sequences in the plasmid library using Bowtie with no mismatches allowed (Langmead, 2009).

The R software package DESeq2 (Love, 2014) was used to evaluate differential gRNA representation in the form of $\log 2$ fold change and p-value between the GFP-high and the GFP-low samples. A robust z-score for each gRNA was calculated using the median and mean-absolute deviation across the $\log 2$ fold changes. To summarize the results at the gene level, the gRNAs are ranked by the robust z-score, and the statistical significances for each gene enriched toward higher rank (RSA up) and the lower rank (RSA down) were evaluated using the Redundant siRNA Activity (RSA) algorithm (König, 2007). Genes with significant RSA up values are candidate targets whose deletion promote resistance to Ferroportin degradation. Conversely, knockout of genes with significant RSA down values may sensitize cells to Hepcidin-induced Ferroportin degradation.

Pathway analysis of screen results was conducted with GeneMANIA software supplemented with literature research (Warde-Farley, 2010).

Validation Experiments 
CRISPR gRNA Lentiviral Packaging and Viral Infection gRNA encoding lentivirus was produced from HEK293T cells using Ready-toUse Lentiviral Packaging Plasmid Mix (Cellecta) and FuGENE6 (Roche) according to standard virus packaging protocol and was used to generate stable cell lines.

Sequences of guide RNAs used in this work: Control (GACCGGAACGATCTCGCGTA); VPS35 (GTAGTGCAGTTCATCAGAAA).

Immunoblotting

Total cell lysates were prepared by direct lysis of cells with RIPA buffer $(50 \mathrm{mM}$ Tris- $\mathrm{HCl}$ at $\mathrm{pH} 7.4,150 \mathrm{mM} \mathrm{NaCl}, 1 \%$ Nonidet P-40, $0.5 \%$ sodium deoxycholate, $0.1 \%$ SDS, 1 mM EDTA, protease inhibitors, phosphatase inhibitors) followed by centrifugation at $13,000 \mathrm{rpm}$ for $15 \mathrm{~min}$ at $4^{\circ} \mathrm{C}$. Equal amount of proteins were resolved by SDS-PAGE, transferred to nitrocellulose membrane, and probed with the indicated antibodies. Primary antibodies included: anti-Ferroportin rabbit polyclonal antibody (Novus Biologicals, Littleton, CO); anti-GAPDH rabbit monoclonal antibody (Cell Signaling Technology 14C10, Danvers, MA). 


\section{Chapter III}

Results

In this study, an inducible HEK293 Ferroportin reporter system suitable for pooled CRISPR screening was established, characterized and screened. The established HEK293::TetOff-FPN1-GFP::Cas9 cell line was confirmed to be sensitive to Hepcidin treatment, and had an appropriate assay effect size (GFP fold change) for pooled CRISPR screening. Investigation of hits by pathway analysis and disease association led to pursuing VPS35 for further validation. Cells with VPS35 knockout demonstrated decreased sensitivity to Hepcidin-induced Ferroportin degradation.

\section{Developing the Ferroportin Pooled CRISPR Screening}

Construction of the HEK293 Ferroportin Reporter Screening System

To build a system for interrogation of Ferroportin regulation, a stable cell line (HEK293::TetOff-FPN1-GFP) expressing human Ferroportin with a C-terminal green fluorescent protein under the control of a Tet-Off inducible promoter was generated (Figure 2). An inducible system was chosen to prevent potential toxicity associated with FPN1 overexpression. Furthermore, Dox could be added at the same time as Hepcidin treatment to pause the transcription of Ferroportin-GFP and allow for identifying regulators specific for degradation as opposed to those that might instead regulate the transcription of new Ferroportin-GFP mRNA transcripts. 


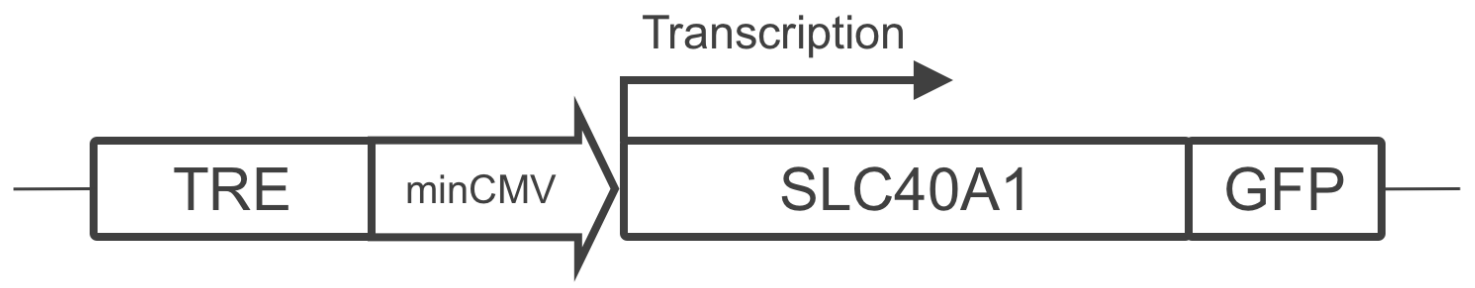

Figure 2. HEK293 Ferroportin (SLC40A1, FPN1)-GFP reporter construct An inducible FPN1-GFP reporter system was built for the investigation of Ferroportin regulators.

Characterization of the FPN1-GFP Reporter System

To confirm the integration of FPN1-GFP reporter into the genome and the correct membrane localization of FPN1-GFP protein, Dox was withdrawn to induce FPN1-GFP expression. After induction, GFP localization could be visualized under fluorescence microscope. Two days of Dox withdrawal was sufficient to induce strong expression of FPN1-GFP, which is localized to the cell surface (Figure 3).

To test for the responsiveness of Ferroportin-GFP reporter to Hepcidin, a single clonal cell line was treated with various doses of Hepcidin and evaluated for FPN1-GFP level by FACS analysis. The established cell line displayed dose-dependent decrease of FPN1-GFP expression in response to Hepcidin treatment and was determined to be suitable for CRISPR screening (Figure 4). The dose of Hepcidin chosen for screening is 1 $\mathrm{ug} / \mathrm{ml}$. 


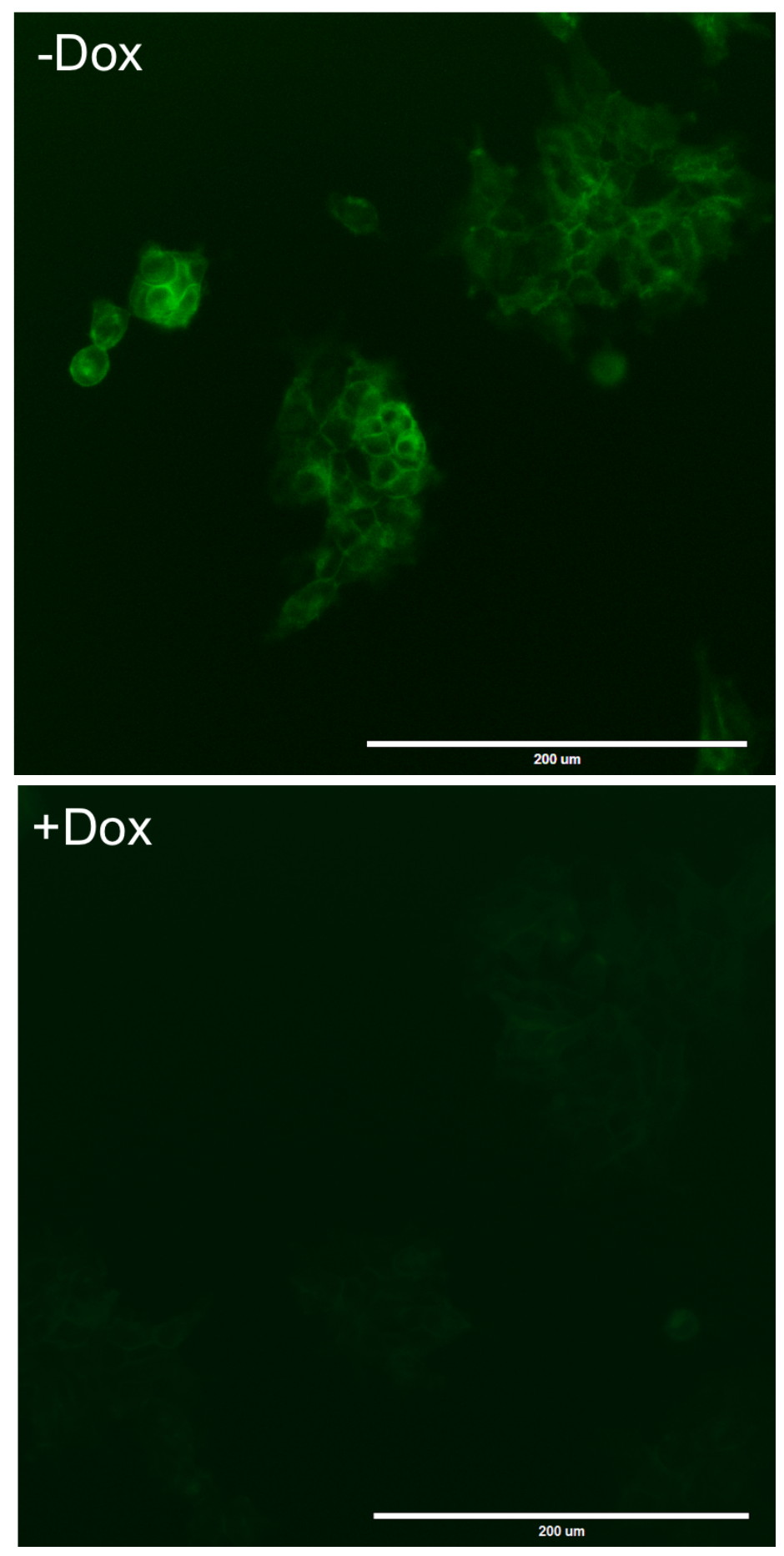

Figure 3. Confocal microscopy image of FPN1-GFP in HEK293.

Abundant GFP fluorescence outlines the surface of HEK293::TetOff-FPN1-GFP after two days of Dox withdrawal. 


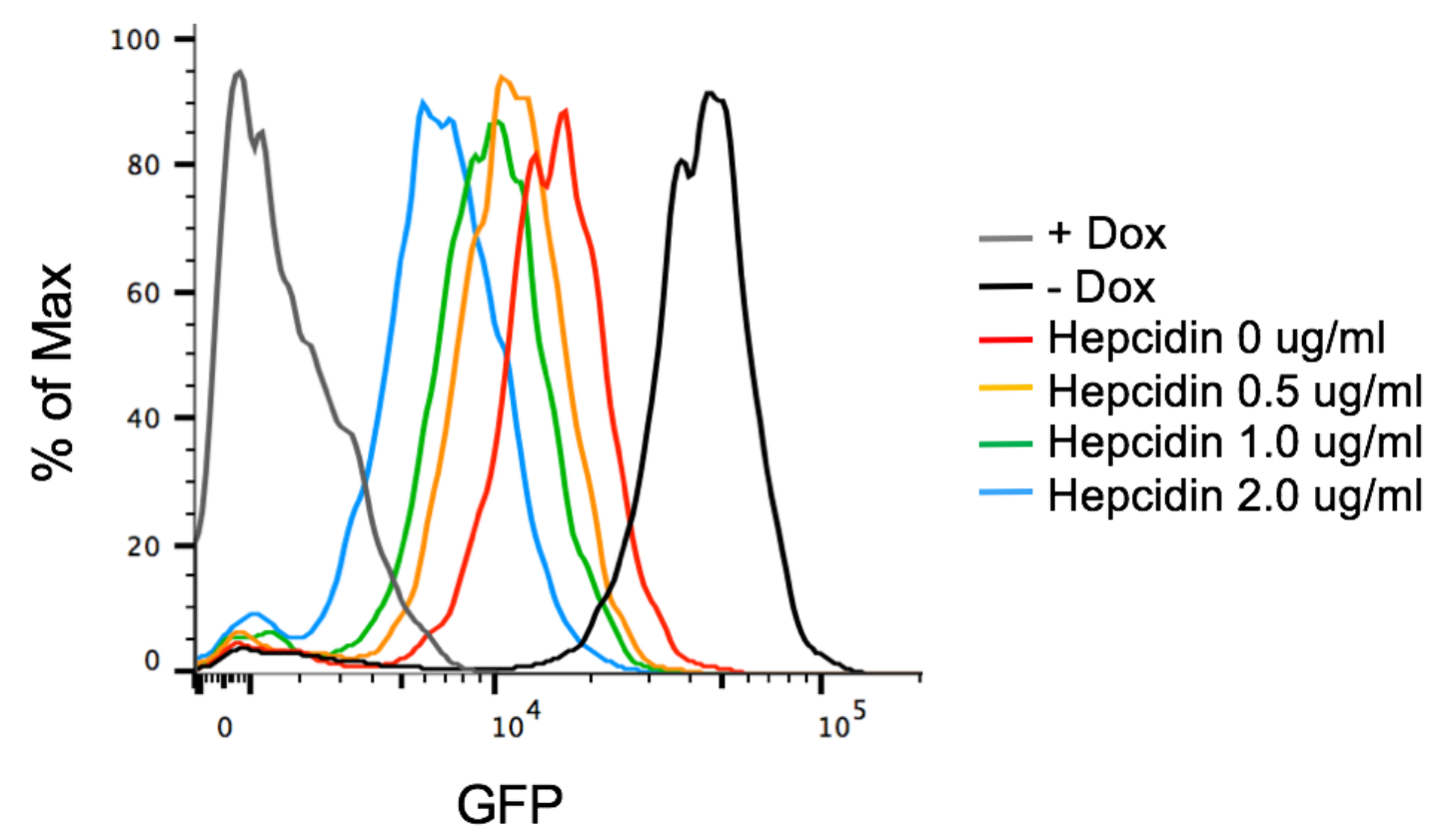

Figure 4. Hepcidin FACS Assay

HEK293::Tet-Off-FPN1-GFP::Cas9 underwent Dox withdrawal for two days to induce FPN1-GFP expression, followed by 24 hour treatment of various dose of Hepcidin. Dox was added at the same time as Hepcidin treatment to halt the induced expression. The black line represents cell with continuous FPN1-GFP induction, serving as the high GFP control; the grey line were cells underwent no induction and served as the low GFP control. Level of GFP detected in Hepcidin treated cells inversely correlated to treatment doses.

Cas9 Characterization and Viral Titer

Following the generation of a single clone of HEK293::Tet-Off-FPN1-GFP cell line, Cas9 was introduced into these cells to enable CRISPR-based gene perturbation. A single clonal population with strong and uniform Cas9 level was picked and expanded for Ferroportin degradation screening (Figure 5). 


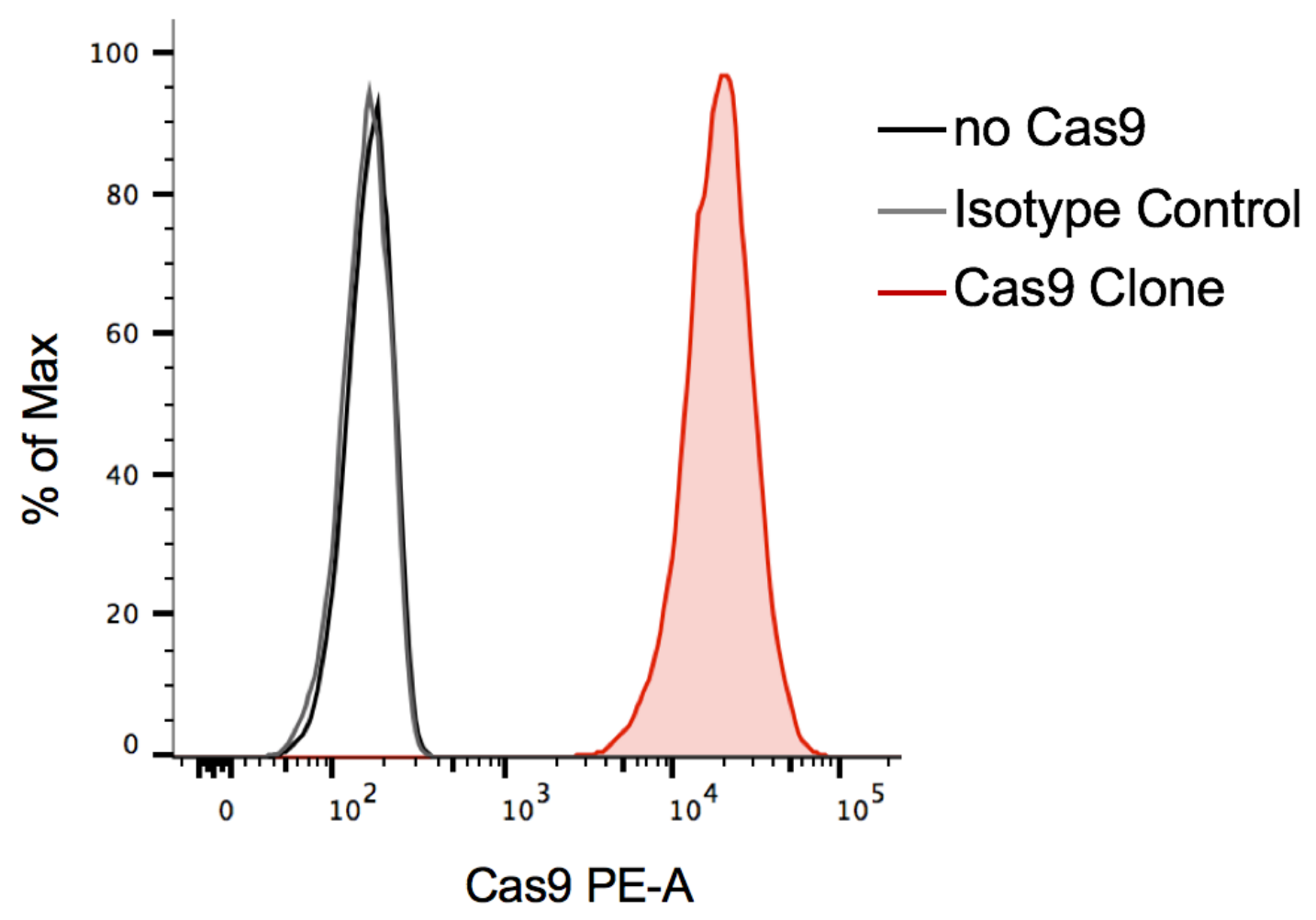

Figure 5. A single clone of HEK293::Tet-Off-FPN1-GFP::Cas9 cells have uniform Cas9 expression.

Cas9 staining of HEK293::Tet-Off-FPN1-GFP::Cas9 cells reveals uniform expression of Cas9 in the cells.

To minimize the probability of co-infecting multiple gRNA constructs into one single cell, it was necessary to control the multiplicity of infection (MOI) during the infection of pooled CRISPR library to HEK293::Tet-Off-FPN1-GFP::Cas9 cells. A viral titer experiment was conducted to determine the amount of pooled CRISPR library virus required for infecting the screening cells at $\mathrm{MOI}=0.5$ in which majority of cells were to be infected by zero or one viral particle. The gRNA expression construct contains a red fluorescence protein (RFP) reporter, thus the infected cells could be identified using 
FACS (Figure 6). The relationship between reference virus volume and \% RFP positive cells were fit with logarithmic model (Figure 7). $6.998 \mu \mathrm{l}$ was identified as the amount of reference virus for MOI of 0.5 on HEK293::FPN1-GFP::Cas9 cells. Amount of pooled library virus for $\mathrm{MOI}=0.5$ was calculated with viral titer multiplier supplied with the pooled CRISPR library.

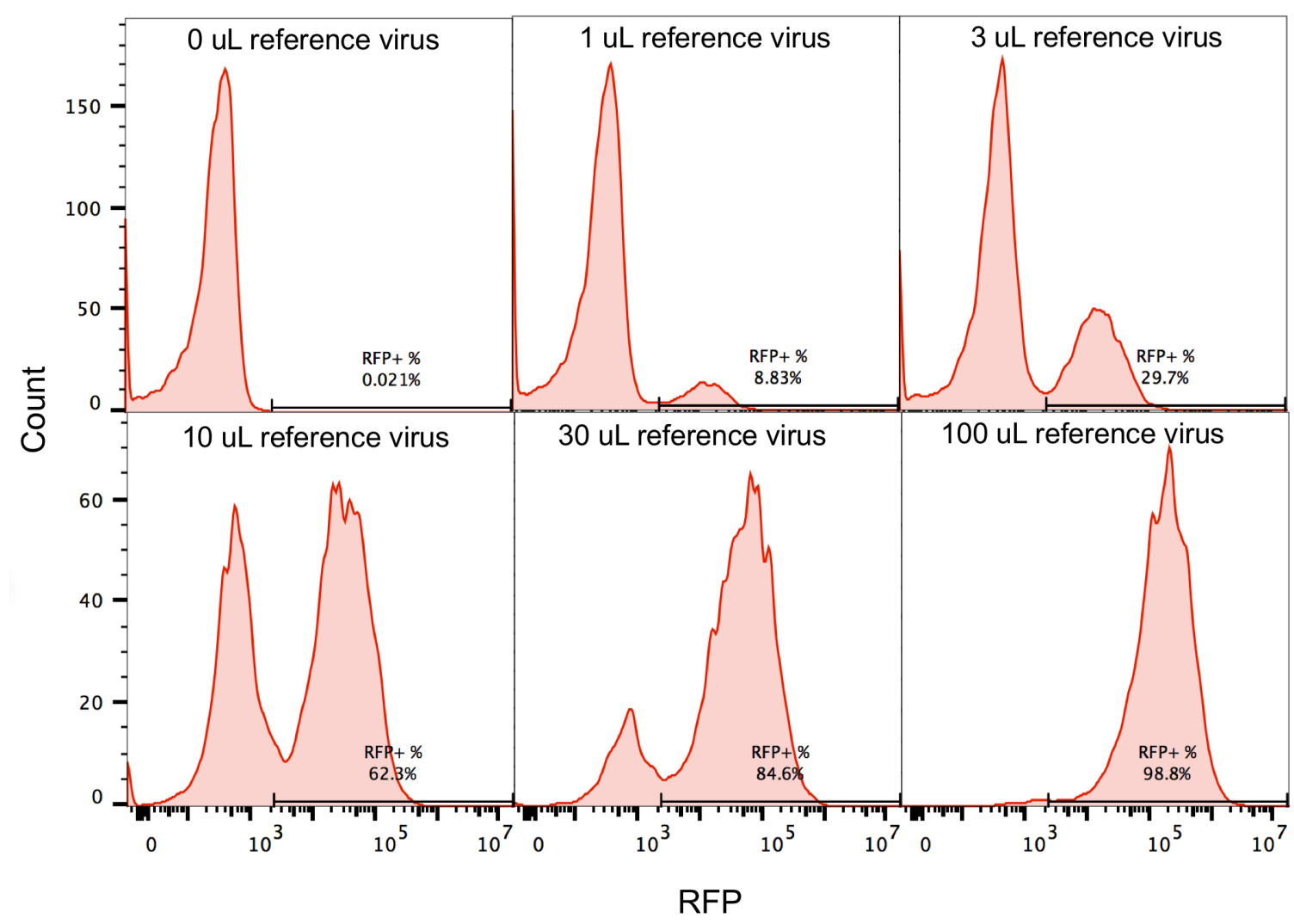

Figure 6. Determining the viral titer for HEK293::Tet-Off-FPN1-GFP::Cas9 - FACS analysis.

Viral titer experiment was performed on HEK293::Tet-Off-FPN1-GFP::Cas9 to determine the amount of CRISPR lentivirus needed to achieve MOI=0.5. Cells were infected with reference virus and measured for percentage of cells with detectable RFP by FACS. 


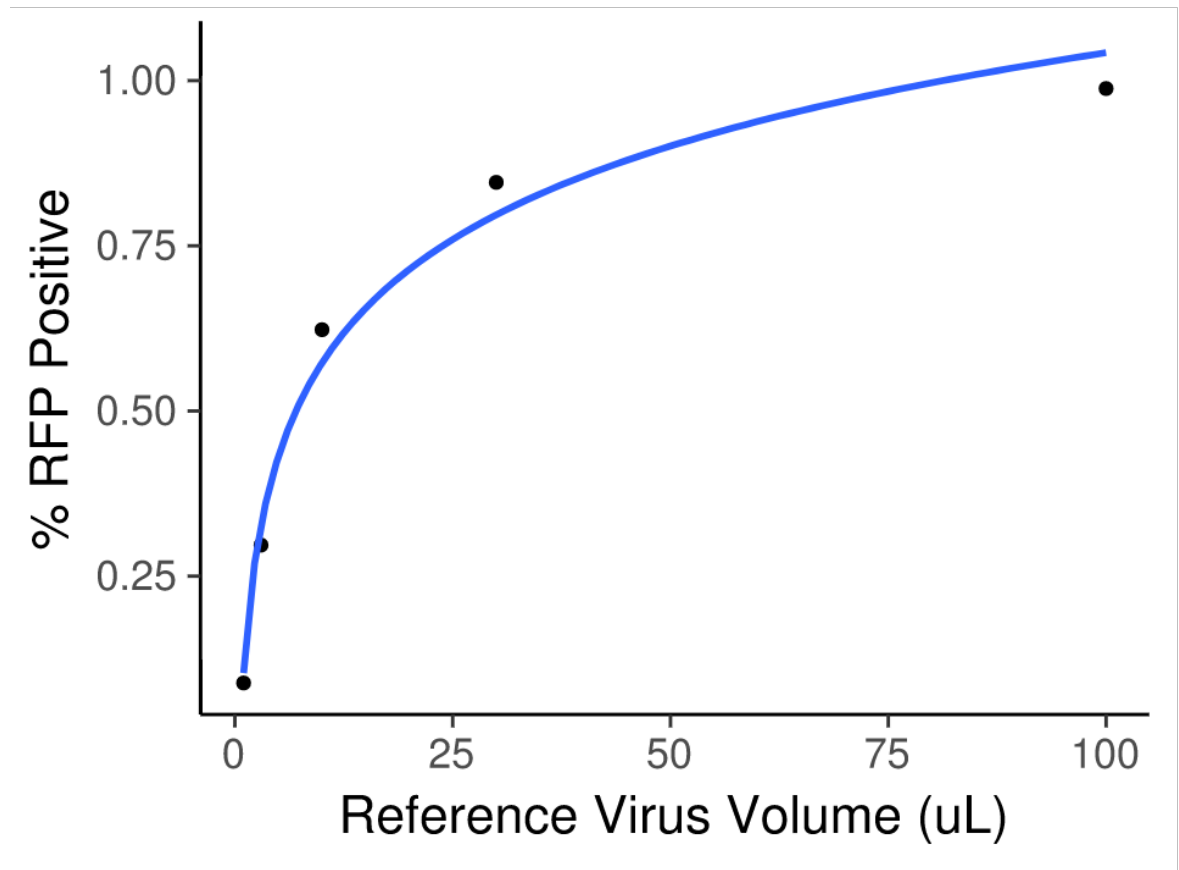

Figure 7. Determining the viral titer for HEK293::Tet-Off-FPN1-GFP::Cas9 - MOI determination.

Viral titer experiment was performed on HEK293::Tet-Off-FPN1-GFP.:Cas9 to determine the amount of CRISPR lentivirus needed to achieve MOI=0.5. The percentage of RFP positive population was mapped against virus volume by logarithmic regression for modeling the infectability of the cells.

The Ferroportin Pooled CRISPR Screen

The Pooled CRISPR Screen

The aim of the screen is to identify molecular mechanisms specific for Hepcidin induced Ferroportin degradation. Therefore, the screen was set up to collect results in both the Hepcidin-treated and -untreated conditions. All pooled CRISPR infected cells underwent two days of Dox withdrawal to induce FPN1-GFP expression. In the treatment 
group, $1 \mu \mathrm{g} / \mathrm{ml}$ Hepcidin was applied in the media for 24 hours prior to harvest. Dox was added to both groups to stop the transcription of FPN1-GFP. Cells of both conditions were sorted by FACS to collect cell subpopulations with $20 \%$ highest and lowest level of GFP. Total of four genomic DNA samples were collected and subjected to Illumina DNA deep sequencing (Figure 8).

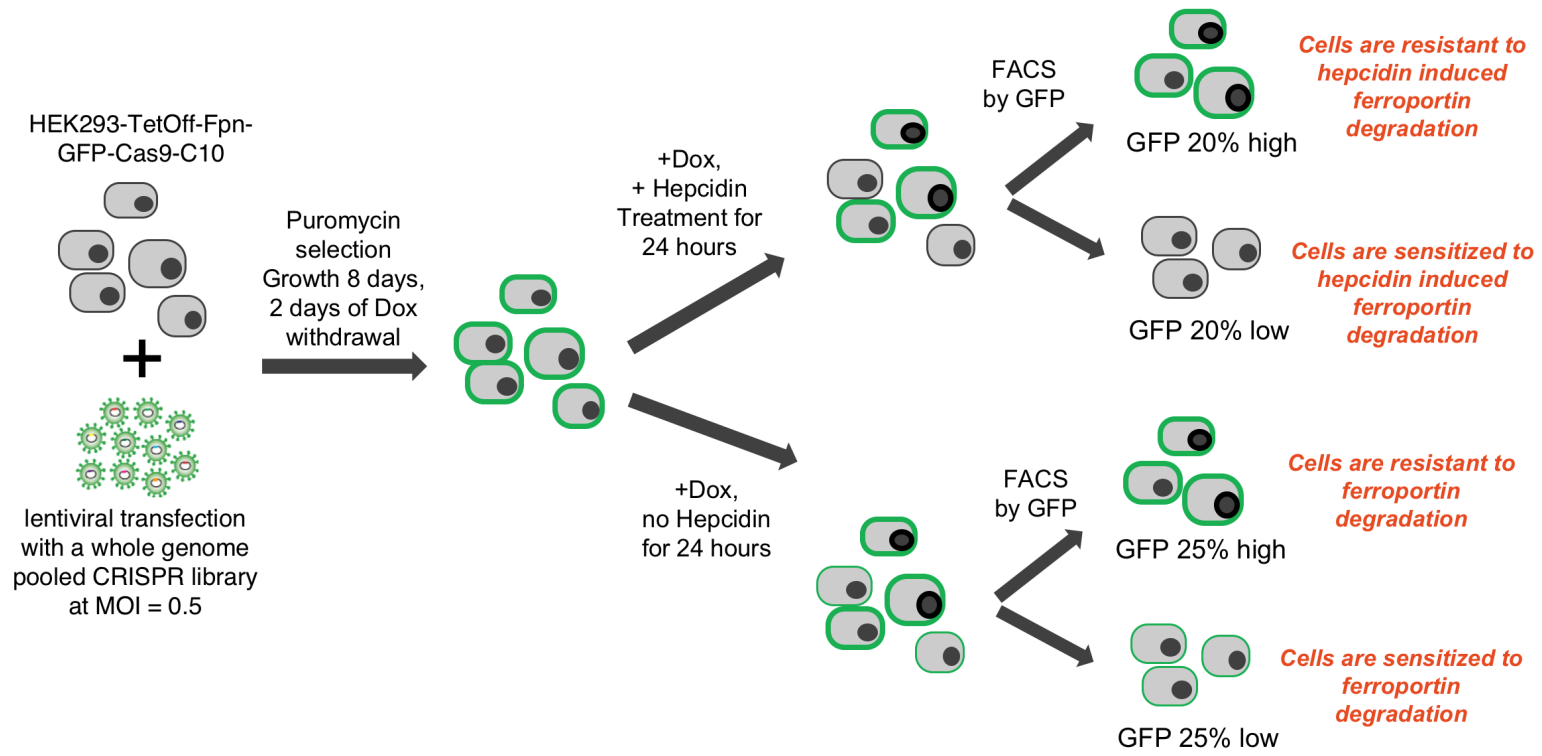

Figure 8. Schematic of Ferroportin-GFP pooled CRISPR screen.

HEK293::Tet-Off-FPN1-GFP::Cas9 cells were transduced with whole genome pooled CRISPR lentiviral libraries and selected by puromycin for stable integration. FACS was used to isolate cell populations based on high or low GFP fluorescence signal. Abundance of $g R N A$ in each subpopulation was quantified by deep sequencing of the gRNA sequence.

\section{Cell Sorting and NGS}

The pooled CRISPR library infected cells underwent sorting by FACS to isolate high FPN1-GFP and low FPN1-GFP populations. The post-sort FACS profile suggested 
the collected populations had distinct GFP levels in either the Hepcidin treated or untreated conditions. The population of cells not stimulated by Hepcidin has an overall higher GFP level comparing to the untreated cells, as expected (Figure 9).

Genomic DNA extracted from the screening cells was first evaluated to have sufficient DNA quantity and purity prior to NGS sequencing. NGS and bioinformatics processing provided gRNA counts of each screening sample for further Quality Control (QC) and statistical analysis. QC of the genomic DNA samples and NGS results confirmed sufficient pooled CRISPR library coverage (Figure 10) and ensured good data quality for statistical analysis of Ferroportin regulators.

\section{Statistical Analysis of the Screening Data}

To identify gRNAs that were unevenly represented in the high FPN1-GFP and low FPN1-GFP populations, a differential analysis was performed for each treatment condition. The differential analysis revealed sufficient screening signal window, with a subset of gRNAs having strong fold differences in representation between the high-GFP and low-GFP populations (Figure 11). These strongly differential gRNAs represent hits from the screen. Different CRISPR gRNAs targeting the same gene may have different target efficiency and produce different degrees of phenotypes due to the position of the mutation on the target gene. For these reasons, the whole genome pooled CRISPR library includes 5 distinct CRISPR gRNAs for every gene. The statistical summarization of the results of the 5 gRNA was used as the primary metric for hit identification and result comparisons. 

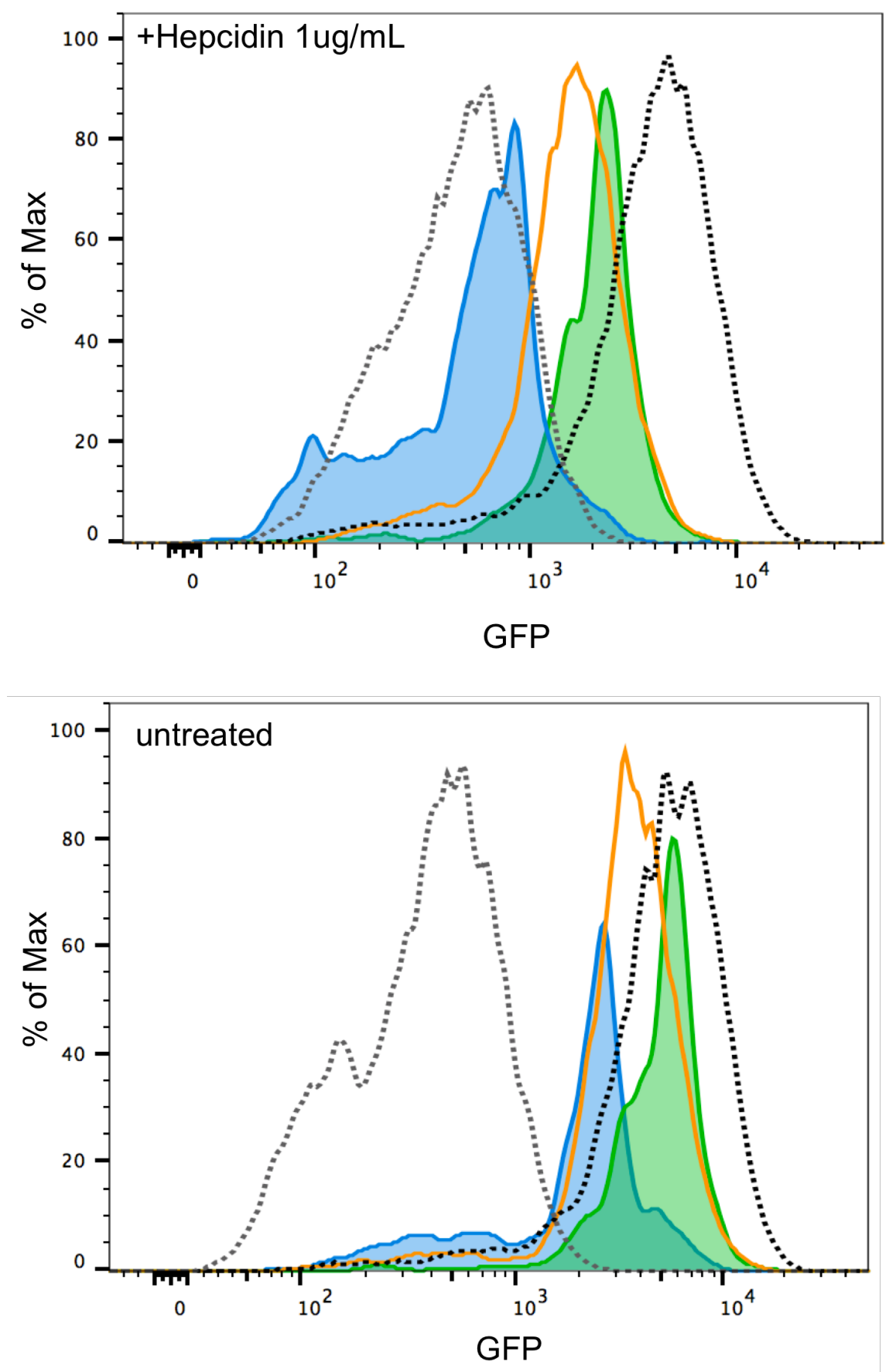

Figure 9. Post-sorting profile for subpopulations from the pooled CRISPR screen FACS.

The FACS profile for pooled CRISPR screen cells reveals successful isolation of high GFP and low GFP populations. Cells in the untreated condition has overall higher level of GFP comparing to the Hepcidin treatment group. The orange peak represents the presort cell population. The blue and green peaks represent the low and high GFP population from the sorting. The black dotted line represents cell with 3 days of 
continuous FPN1-GFP induction, serving as the high GFP control; the grey dotted line represents cells underwent no induction and served as the low GFP control.

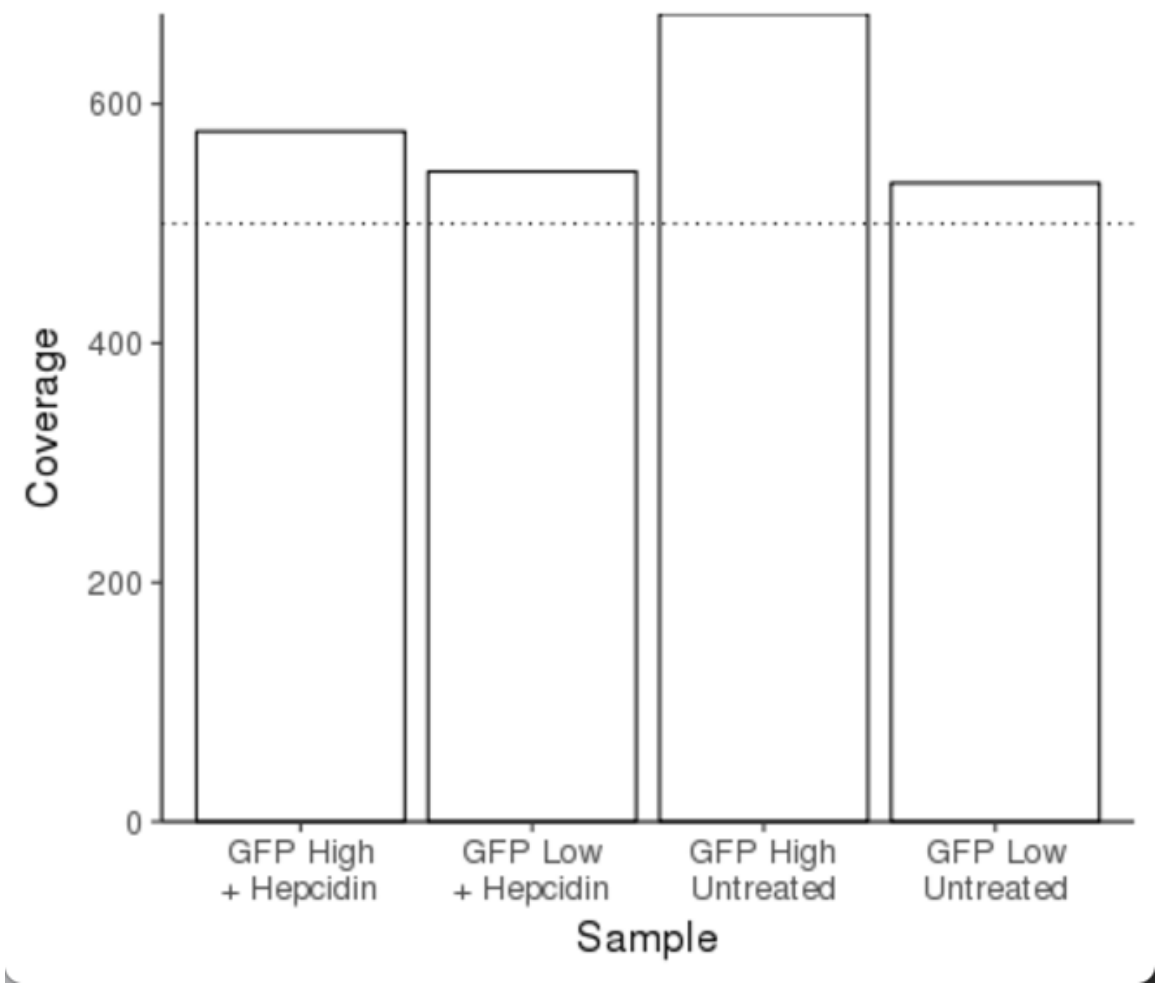

Figure 10.NGS and Quality Control for Pooled CRISPR Screen Results.

Sufficient quantities of NGS reads were obtained from the screening samples. The coverage of the pooled CRISPR library gRNA was over 500-fold in the sequencing result. 


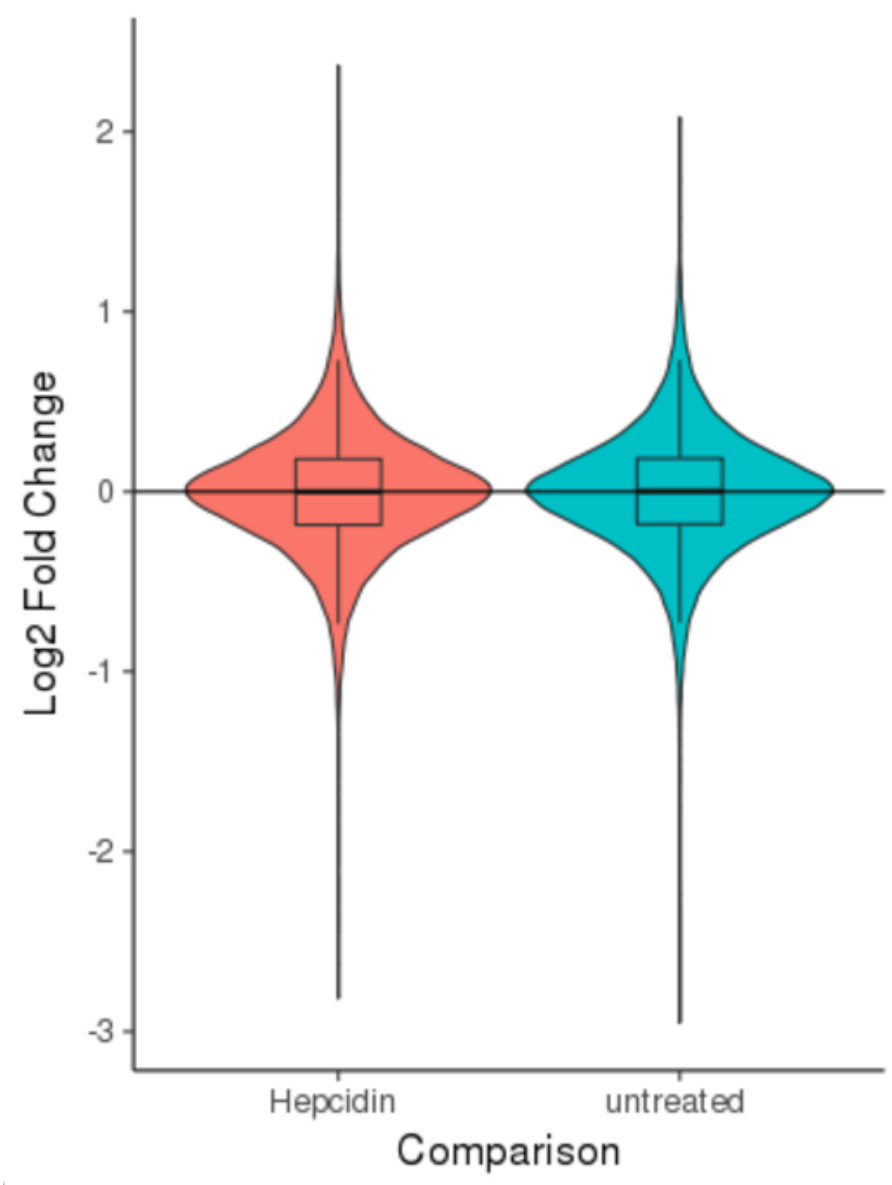

Figure 11.The distribution of the differential gRNA representation.

The distribution of differential representation of $g R N A$ between the FPNI-GFP high and low populations in both the Hepcidin treated and untreated condition revealed similar signal window between the two screening conditions and sufficient signal for statistical analysis.

Ferroportin Pooled CRISPR Screen Hit Selection and Validation

To confirm the performance of the screen, the results of Ferroportin-targeting gRNAs were first reviewed. Four of the five gRNAs designed to target Ferroportin had consistent negative fold changes of $<-1.5$ while one gRNA did not affect FPN1-GFP level. Further investigation revealed that the CRISPR cut site of this ineffective gRNA 
was located outside of the exon regions of Ferroportin. The RSA method summarizes the performance of the 5 gRNAs against each gene by comparing the ranking of all 5 gRNAs or their subset against the remaining population. As expected, Ferroportin gRNAs were significantly overrepresented in the GFP-low population in both the Hepcidin treated and untreated experiments, providing further confidence that the analytical method appropriately summarized the activities of the $5 \mathrm{gRNAs}$ and provided resistance to outlier data points (Figure 12).

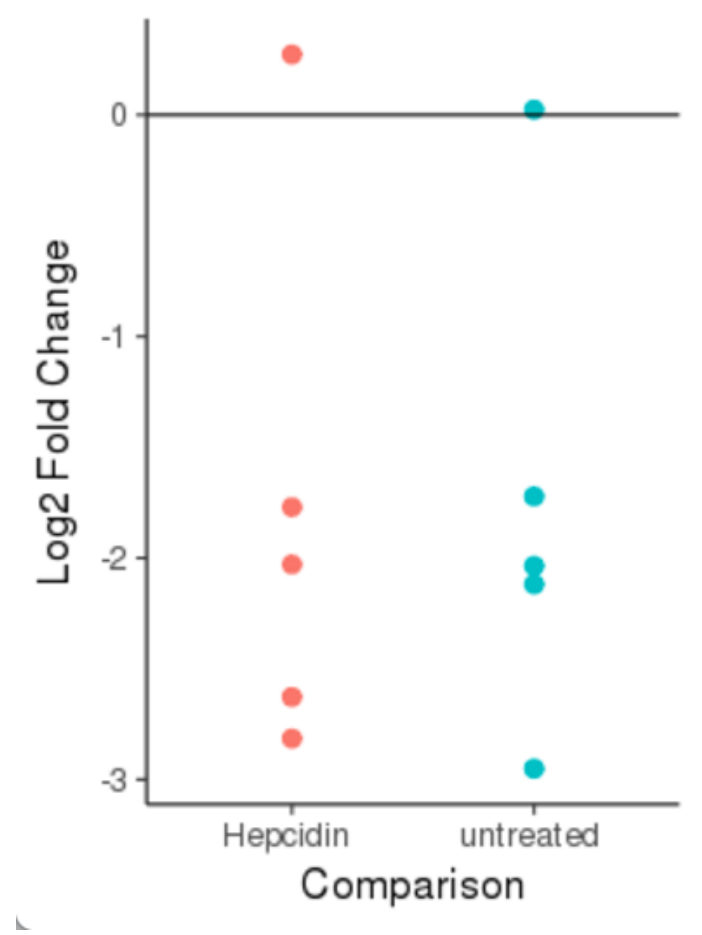

Figure 12. Ferroportin gRNAs were under-represented in the high FPN1-GFP subpopulation.

gRNAs against Ferroportin (SLC40A1, FPN1) were under-represented in the high FPN1GFP population, confirming the effective sorting of high FPN1-GFP and low FPN1-GFP cell populations by FACS. Four out of five gRNAs targeting Ferroportin had strong negative fold changes when comparing the gRNA representation in GFP high vs low populations. 
Transport Related Pathways Regulate Ferroportin-GFP level

The screen analysis results allowed for investigation of both the positive and negative regulators of FPN1-GFP. RSA down was used as the matrix for investigating positive regulators of FPN1-GFP membrane localization and stability, highlighting genes that upon knockout had reduced FPN1-GFP signal. Comparing the results of Hepcidin treated and untreated experiments, the two conditions shared strikingly similar positive regulators (Figure 13). The shared hits largely consist of genes involved in vesicle transport. It is likely the vesicle transport machinery is important for the localization of Ferroportin to the plasma membrane. Ferroportin that fails to localize to the plasma membrane are likely degraded in the presence or absence of Hepcidin.

The RSA up result identified negative regulators of the FPN1-GFP signal. The genes with significant RSA up results in Hepcidin treated and untreated experiments have little overlap, suggesting that Hepcidin induced Ferroportin degradation may have distinct molecular mechanism separated from the routine turnover of Ferroportin (Figure 14). Further investigation of the 65 genes that were uniquely significant in the Hepcidin treated condition but not in the untreated condition revealed pathways associated with targeted protein degradation including the endosomal sorting complex required for transport (ESCRT) pathway, peroxisome biogenesis genes and the Retromer Complex (Figure 15). Overall, transport related pathways emerge as the important regulators of Ferroportin level in the context of Hepcidin-induced degradation. 


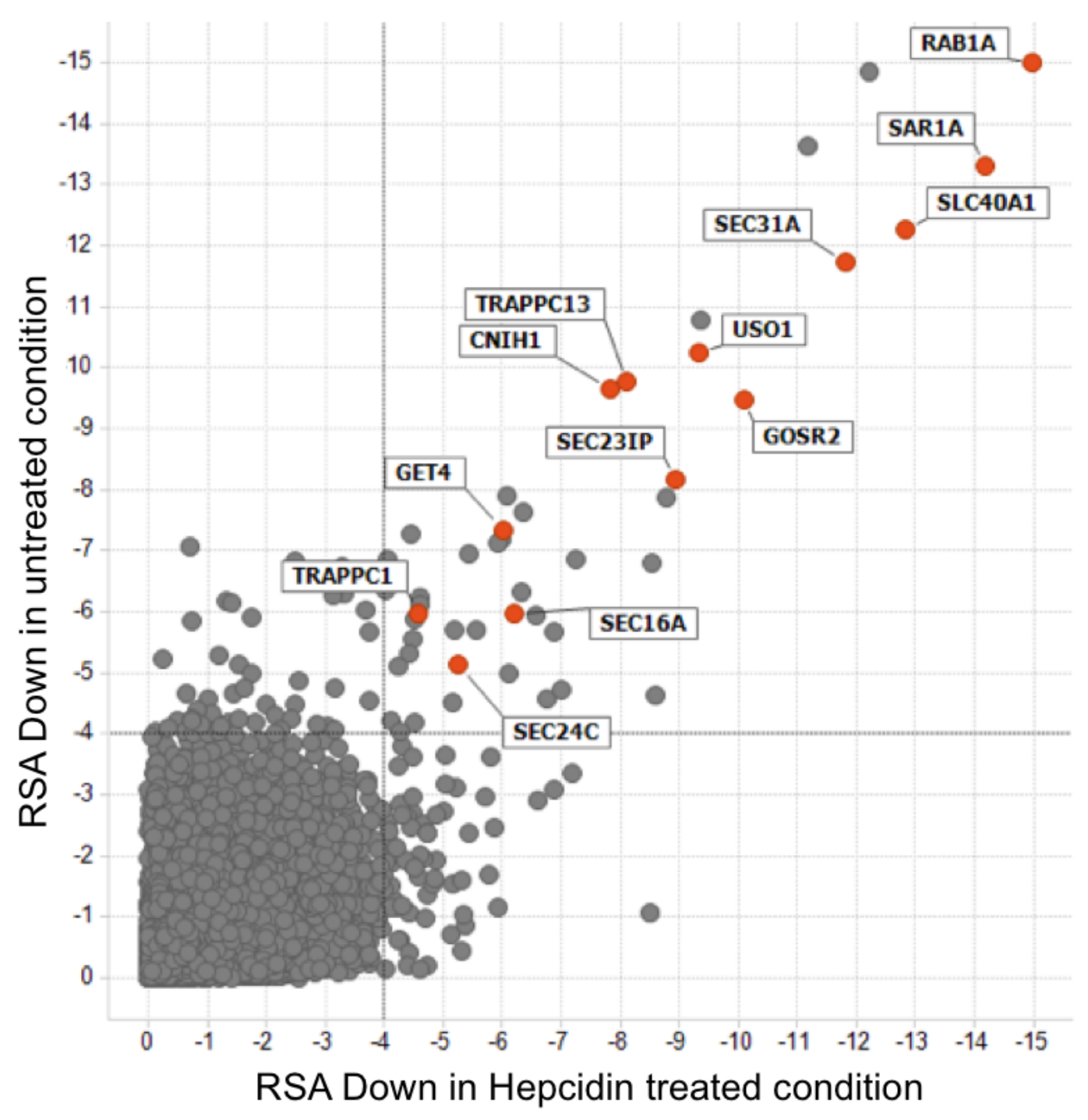

Figure 13. Positive regulators of Ferroportin identified from the pooled CRISPR screen.

The RSA Down statistical metric highlighted positive regulators of FPN1-GFP, in which CRISPR knockout of indicated genes reduced FPN1-GFP signal. Ferroportin (SLC40A1) and vesicle transport-related genes are highlighted in red. 


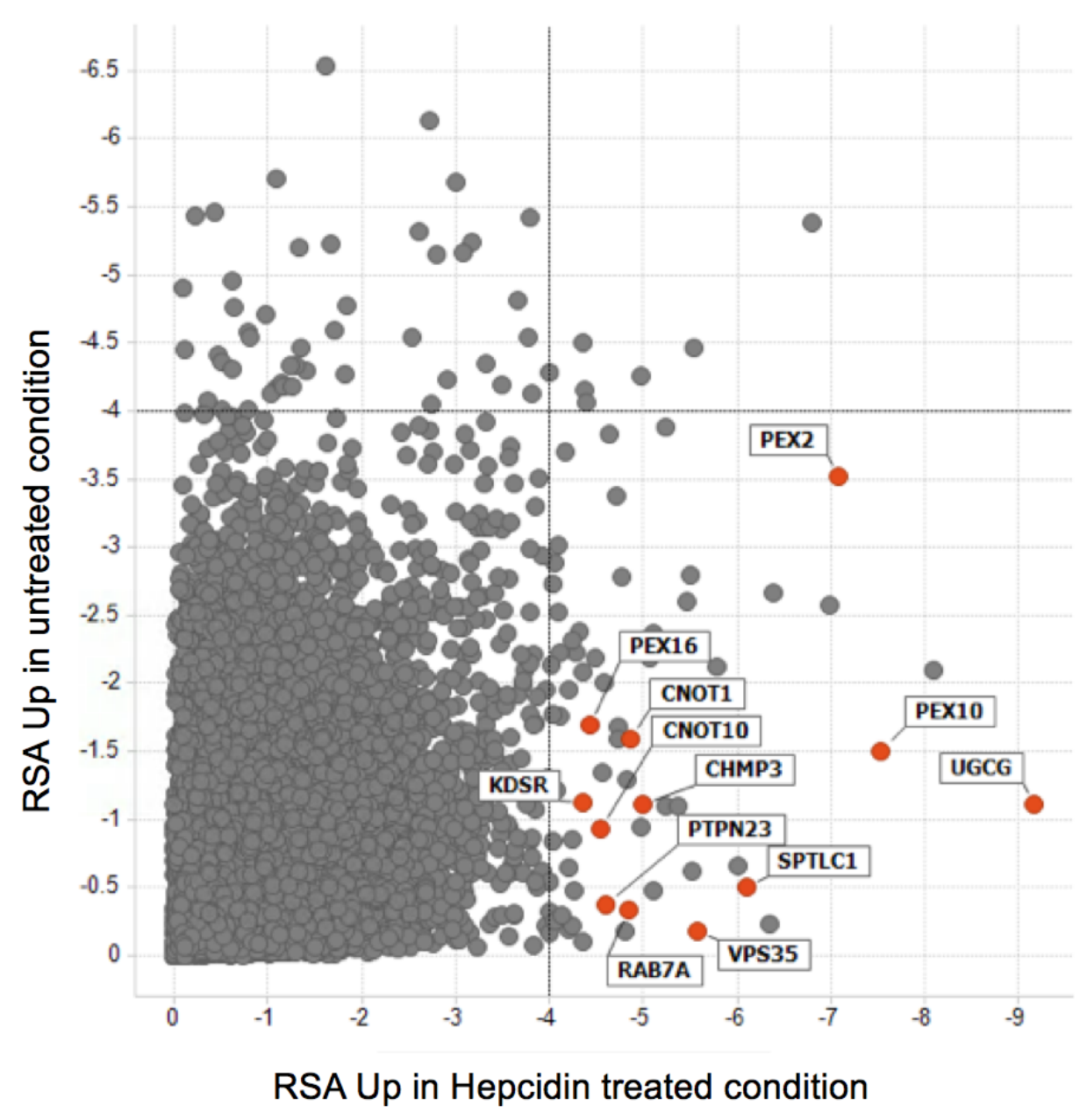

Figure 14. Negative regulators of Ferroportin identified from the pooled CRISPR screen.

Comparison of negative regulators of Ferroportin in Hepcidin treated condition and untreated condition. Genes of pathways enriched in the Hepcidin condition are highlighted in red. 


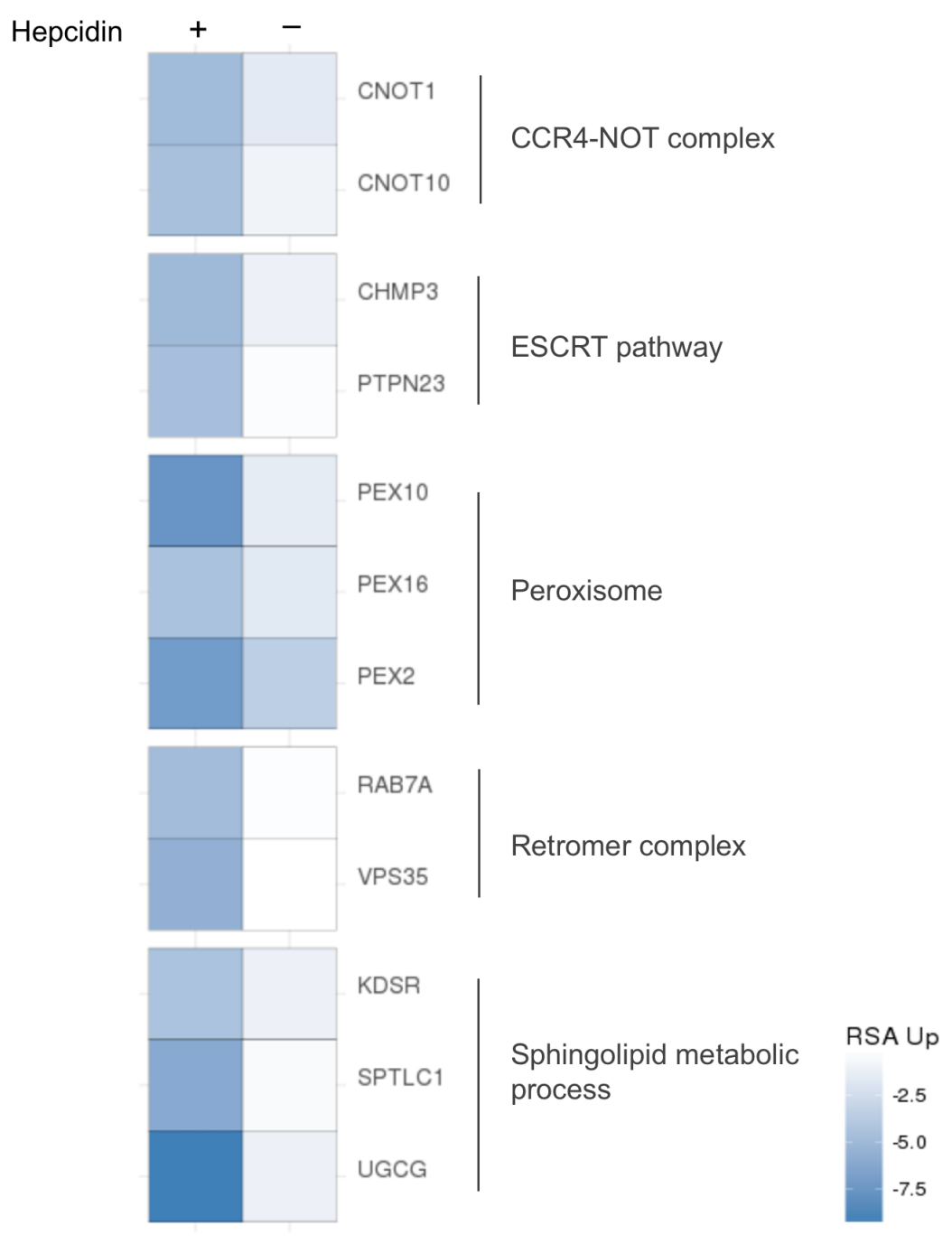

Figure 15. Pathways specifically enriched in the Hepcidin treated condition.

VPS35 is Important for Hepcidin-mediated Degradation of Ferroportin

For validation, the Retromer Complex was selected as it demonstrates a striking specificity in the primary screen and it has reportedly known function in the transport of specific transmembrane proteins. Two screen hits are associated with the Retromer Complex, Ras-Associated Protein RAB7A (RAB7A) and Vacuolar Protein SortingAssociated Protein 35 (VPS35). VPS35 was selected for further studies as it is a core 
member of the Retromer Complex while RAB7A is reportedly involved in other cellular and physiological events (Ceresa, 2006; Edinger, 2003). Stable cell lines from HEK293::Tet-Off-FPN1-GFP::Cas9 with VPS35 knockout or non-targeting control were established using the top performing gRNA for VPS35 from the screen and a nontargeting control gRNA.

In the validation FACS assay, VPS35 knockout cells displayed strong reduction of response to Hepcidin-induced FPN1-GFP degradation in comparison to the control gRNA infected cells, confirming the primary screen result (Figure 16; Table 1). Western blot with Ferroportin antibody further confirmed the observation (Figure 17). Together, these results suggest that VPS35 is involved in Hepcidin-mediated Ferroportin degradation and that in the wild-type setting, it aids Hepcidin degradation of Ferroportin.
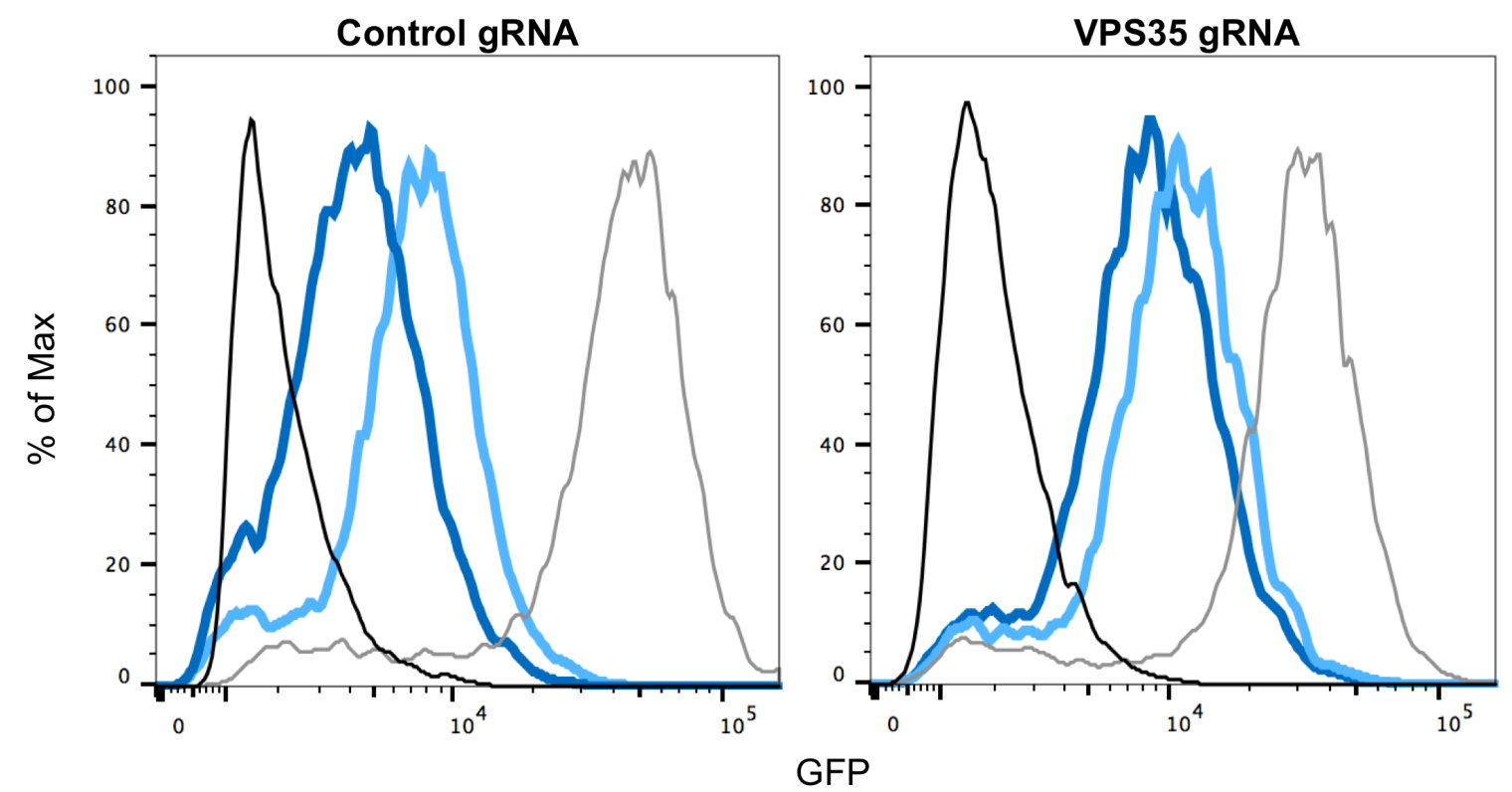


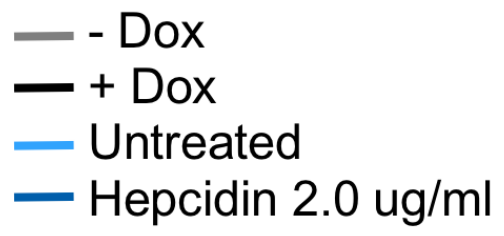

Figure 16. VPS35 knockout cells have reduced sensitivity to Hepcidin - FACS

validation.

HEK293::Tet-Off-FPN1-GFP::Cas9 expressing VPS35 gRNA or non-targeting control $g R N A$ were tested for responsiveness to Hepcidin treatment. Cells underwent Dox withdrawal for two days to induce FPN1-GFP expression, followed by 24 hour treatment of $2 \mathrm{ug} / \mathrm{ml}$ Hepcidin. Dox was added at the same time as Hepcidin treatment to halt Ferroportin transcription. The grey line represents cell with continuous FPN1-GFP induction, serving as the high GFP control; the black line represents cells with no induction, serving as the low GFP control.

Table 1. VPS35 knockout cells have reduced sensitivity to Hepcidin - GFP quantitation.

gRNA Untreated Hepcidin treated Decrease of median GFP value in Hepcidin treated condition comparing to untreated

$\begin{array}{llll}\text { VPS35 } & 10787 & 8465 & 21.5 \%\end{array}$

Control $7360 \quad 4283 \quad 41.8 \%$

Median FPN1-GFP value with or without Hepcidin treatment in cells expressing control gRNA or VPS35 $g R N A$. 

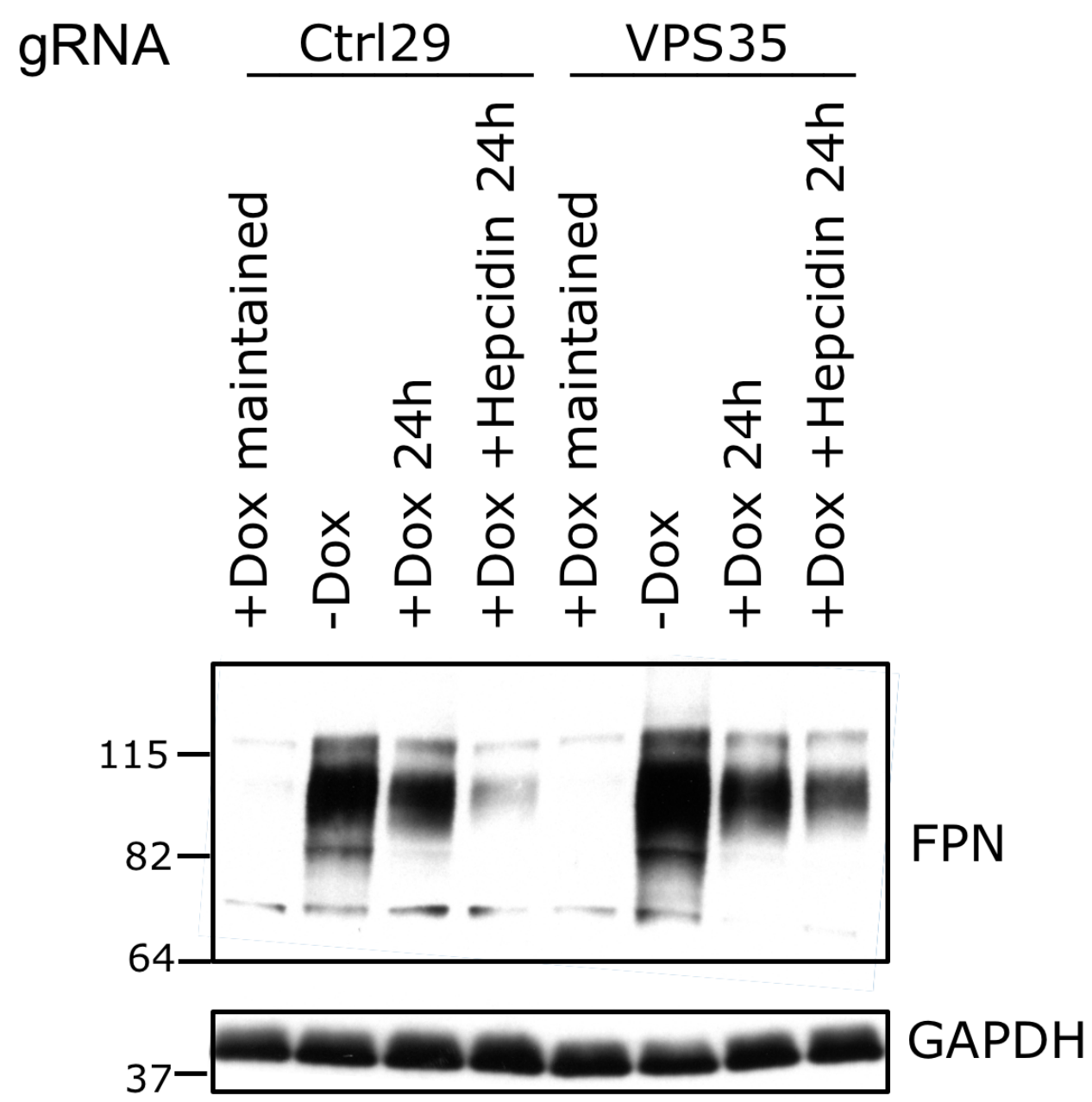

Figure 17. Western blot analysis of Ferroportin-GFP expression upon Hepcidin treatment.

Knockout of VPS35 by CRISPR reduced Hepcidin-dependent degradation. Cell lysates were fractionated by SDS PAGE and blotted with anti-FPN1 antibody. 


\section{Chapter IV}

Discussion

Iron is essential for normal physiological function of living organisms, and our understanding of iron homeostasis is incomplete. Hepcidin-induced degradation of Ferroportin and its importance in regulation of circulatory iron are well established, yet the molecular mechanism underlying Hepcidin-induced degradation of Ferroportin is largely unknown. Previous work suggested that ubiquitination of Ferroportin is necessary for Hepcidin-mediated rapid degradation of Ferroportin, but no specific gene or pathway has been identified (Ross, 2014; Qiao, 2014). Here a functional genetic screening approach allows for unbiased study of the Hepcidin-induced Ferroportin degradation and leads to the identification of VPS35 as an important regulator for Hepcidin-mediated degradation of Ferroportin.

Review of the screen hits suggests that the mechanism for the Hepcidin-mediated degradation of Ferroportin is distinct from its regular protein turnover. Hepcidin-induced degradation of Ferroportin is a rapid process and is believed to be a host-defense mechanism against pathogens, in which an accelerated response quickly depletes iron available to pathogens in the circulation and limits pathogen growth (Rivera, 2005; Ganz, 2015). It is likely that Hepcidin-induced rapid turnover of Ferroportin is a resource consuming process reserved for host defense and not utilized in the normal turnover of Ferroportin.

Investigation of screening hits that are specific for Hepcidin-induced Ferroportin degradation leads to the hypothesis that VPS35, a component of the Retromer Complex, functions as a regulator of iron homeostasis. Interestingly, VPS35 dysfunction has been 
linked to familial Parkinson's disease. Specifically, mutations in VPS35 are associated with development of autosomal-dominant, late-onset Parkinson's disease (Vilariño-Güell 2011; Zimprich, 2011; Zavodszky, 2014). Dysfunction of VPS35 and the other Retromer Complex members also reportedly contribute to core pathological features of the Alzheimer disease (Small, 2005). In both diseases, iron accumulation has been implicated in the pathology and pathogenesis (Good, 1992; Martin, 2008; Wang, 2016; Ayton, 2015). Interestingly, dysregulation of Hepcidin has also been observed in Parkinson patients (Raha, 2013). VPS35 involvement in iron regulation inferred from this study is consistent with the reported disease relevance of VPS35 and iron elevation implicated in the pathogenesis of the two progressive neurodegenerative diseases. This finding is the first report that directly connects VPS35 to iron homeostasis through the HepcidinFerroportin pathway.

The Retromer Complex is regarded as an important element of the endosomal sorting machinery (Seaman, 2004). Concurrently, the endosomal sorting complex required for transport (ESCRT) pathway members are also among the hits in this study. Crosstalk of retromer and ESCRT components have been reported but the exact mechanisms has not been determined (Strochlic, 2008). VPS35 is a member of the cargorecognition module within the Retromer Complex which reportedly binds to specific transmembrane proteins and the complex undergoes directed transport mediated by the tabulation module of the Retromer Complex (Small, 2015). Retromer has been shown to regulate physiological processes through mediating the correct localization and/or the uptake and recycling of specific transmembrane proteins (Bonifacino, 2008; Pocha, 2011; Steinberg, 2013). Further studies are required to dissect the mechanistic detail of VPS35 
and endosome cycling for Ferroportin degradation, including clarification of whether the Retromer Complex directly interacts with Ferroportin to regulate Ferroportin degradation or through indirect mechanisms.

The exact mechanism of how VPS35 regulates Ferroportin degradation remains to be investigated. In the validation efforts, CRISPR editing of VPS35 did not fully abolish Hepcidin induced degradation. Several possibilities are hypothesized for this observation: 1) Disease-associated point mutations or CRISPR induced mutation in VPS35 may have only partially abolished VPS35 function; 2) Ferroportin degradation could be context specific and regulated by additional factors beyond VPS35 and the retromer; 3) A molecular mechanism exists that is redundant to the Retromer Complex. Additional experiments by knocking out additional members of the Retromer Complex in combination with other top hits from the screen may clarify regulatory mechanisms of Hepcidin-induced Ferroportin degradation. Testing additional cell types may also provide further insight on the context specificity of Ferroportin regulation, as their cellular composition may be distinct enough from HEK293. Interestingly, GTEx profile reveals that VPS35 has significantly lower level of expression in liver, an organ important for iron storage, comparing to other organs and tissues (Lonsdale, 2013).

In summary, the Ferroportin whole genome pooled CRISPR screen result lead to the discovery of VPS35, a member of the Retromer Complex, as an important regulator of the Hepcidin-mediated Ferroportin degradation. Diseases genetically linked to the dysfunction of Retromer Complex, along with its iron overload phenotype, further suggest the relevance of Retromer Complex in iron homeostasis. Continued mechanistic studies are necessary for understanding how VPS35 and the Retromer Complex regulate 
Ferroportin degradation and iron homeostasis, potentially providing insights and therapeutic opportunities for the Parkinson's and the Alzheimer disease population. 


\section{References}

Ayton, S., Faux, N. G., Bush, A. I., Weiner, M. W., Aisen, P., Petersen, R., ... \& Beckett, L. (2015). Ferritin levels in the cerebrospinal fluid predict Alzheimer's disease outcomes and are regulated by APOE. Nature communications, 6, ncomms 7760 .

Bonifacino, J. S., \& Hurley, J. H. (2008). Retromer. Current opinion in cell biology, 20(4), 427-436.

Cemonesi, L., Forni, G. L., Soriani, N., Lamagna, M., Fermo, I., Daraio, F., ... \& Camaschella, C. (2005). Genetic and clinical heterogeneity of Ferroportin disease. British journal of haematology, 131(5), 663-670.

Ceresa, B. P., \& Bahr, S. J. (2006). rab7 activity affects epidermal growth factor: epidermal growth factor receptor degradation by regulating endocytic trafficking from the late endosome. Journal of Biological Chemistry, 281(2), 1099-1106.

Chen, B., Gilbert, L. A., Cimini, B. A., Schnitzbauer, J., Zhang, W., Li, G. W., ... \& Huang, B. (2013). Dynamic imaging of genomic loci in living human cells by an optimized CRISPR/Cas system. Cell, 155(7), 1479-1491.

Donovan, A., Lima, C. A., Pinkus, J. L., Pinkus, G. S., Zon, L. I., Robine, S. and Andrews, N. C. (2005). The iron exporter Ferroportin/Slc40a1 is essential for iron homeostasis. Cell metabolism, 1(3),191-200.

Drakesmith, H., Schimanski, L. M., Ormerod, E., Merryweather-Clarke, A. T., Viprakasit, V., Edwards, J. P., ... \& Robson, K. J. (2005). Resistance to hepcidin is conferred by hemochromatosis-associated mutations of ferroportin. Blood, 106(3), 1092-1097.

Edinger, A. L., Cinalli, R. M., \& Thompson, C. B. (2003). Rab7 prevents growth factorindependent survival by inhibiting cell-autonomous nutrient transporter expression. Developmental cell, 5(4), 571-582.

Ganz, T., \& Nemeth, E. (2015). Iron homeostasis in host defense and inflammation. Nature reviews Immunology, 15(8), 500-510.

Good, P. F., Olanow, C. W., \& Perl, D. P. (1992). Neuromelanin-containing neurons of the substantia nigra accumulate iron and aluminum in Parkinson's disease: a LAMMA study. Brain research, 593(2), 343-346. 
König, R., Chiang, C. Y., Tu, B. P., Yan, S. F., DeJesus, P. D., Romero, A., ... \& Chanda, S. K. (2007). A probability-based approach for the analysis of large-scale RNAi screens. Nature methods, 4(10), 847-849.

Langmead, B., Trapnell, C., Pop, M., \& Salzberg, S. L. (2009). Ultrafast and memoryefficient alignment of short DNA sequences to the human genome. Genome biology, 10(3), 1.

Lonsdale, J., Thomas, J., Salvatore, M., Phillips, R., Lo, E., Shad, S., ... \& Foster, B. (2013). The genotype-tissue expression (GTEx) project. Nature genetics, 45(6), $580-585$.

Love, M. I., Huber, W. and Anders, S. (2014). Moderated estimation of fold change and dispersion for RNA-seq data with DESeq2. Genome biology, 15(12), 1.

Martin, W. W., Wieler, M., \& Gee, M. (2008). Midbrain iron content in early Parkinson disease A potential biomarker of disease status. Neurology, 70 (16 Part 2), 14111417.

Montosi, G., Donovan, A., Totaro, A., Garuti, C., Pignatti, E., Cassanelli, S., ... \& Pietrangelo, A. (2001). Autosomal-dominant hemochromatosis is associated with a mutation in the Ferroportin (SLC11A3) gene. The Journal of clinical investigation, 108(4), 619-623.

Nemeth, E., Tuttle, M. S., Powelson, J., Vaughn, M. B., Donovan, A., Ward, D. M., ... \& Kaplan, J. (2004). Hepcidin regulates cellular iron efflux by binding to Ferroportin and inducing its internalization. Science, 306(5704), 2090-2093.

Njajou, O. T., Vaessen, N., Joosse, M., Berghuis, B., van Dongen, J. W., Breuning, M. H., ... \& van Duijn, C. M. (2001). A mutation in SLC11A3 is associated with autosomal dominant hemochromatosis. Nature genetics, 28(3).

Pietrangelo, A., Montosi, G., Totaro, A., Garuti, C., Conte, D., Cassanelli, S., ... \& Gasparini, P. (1999). Hereditary hemochromatosis in adults without pathogenic mutations in the hemochromatosis gene. New England Journal of Medicine, $341(10), 725-732$.

Pocha, S. M., Wassmer, T., Niehage, C., Hoflack, B., \& Knust, E. (2011). Retromer controls epithelial cell polarity by trafficking the apical determinant Crumbs. Current Biology, 21(13), 1111-1117.

Qiao, B., Sugianto, P., Fung, E., del-Castillo-Rueda, A., Moran-Jimenez, M. J., Ganz, T. \& Nemeth, E. (2012). Hepcidin-induced endocytosis of Ferroportin is dependent on Ferroportin ubiquitination. Cell metabolism, 15(6), 918-924.

Raha, A. A., Vaishnav, R. A., Friedland, R. P., Bomford, A., \& Raha-Chowdhury, R. (2013). The systemic iron-regulatory proteins hepcidin and ferroportin are 
reduced in the brain in Alzheimer's disease. Acta neuropathologica communications, 1(1), 55.

Rivera, S., Nemeth, E., Gabayan, V., Lopez, M. A., Farshidi, D., \& Ganz, T. (2005). Synthetic hepcidin causes rapid dose-dependent hypoferremia and is concentrated in ferroportin-containing organs. Blood, 106(6), 2196-2199.

Ross, S. L., Tran, L., Winters, A., Lee, K. J., Plewa, C., Foltz, I., ... \& Cooke, K. S. (2012). Molecular mechanism of hepcidin-mediated ferroportin internalization requires ferroportin lysines, not tyrosines or JAK-STAT. Cell metabolism, 15(6), 905-917.

Seaman, M. N. (2004). Cargo-selective endosomal sorting for retrieval to the Golgi requires retromer. J Cell Biol, 165(1), 111-122.

Sham, R. L., Phatak, P. D., West, C., Lee, P., Andrews, C., \& Beutler, E. (2005). Autosomal dominant hereditary hemochromatosis associated with a novel ferroportin mutation and unique clinical features. Blood Cells, Molecules, and Diseases, 34(2), 157-161.

Sham, R. L., Phatak, P. D., Nemeth, E., \& Ganz, T. (2009). Hereditary hemochromatosis due to resistance to hepcidin: high hepcidin concentrations in a family with C326S ferroportin mutation. Blood, 114(2), 493-494.

Small, S. A., Kent, K., Pierce, A., Leung, C., Kang, M. S., Okada, H., ... \& Kim, T. W. (2005). Model-guided microarray implicates the retromer complex in Alzheimer's disease. Annals of neurology, 58(6), 909-919.

Small, S. A., \& Petsko, G. A. (2015). Retromer in Alzheimer disease, Parkinson disease and other neurological disorders. Nature Reviews. Neuroscience, 16(3), 126.

Steinberg, F., Gallon, M., Winfield, M., Thomas, E., Bell, A. J., Heesom, K. J., ... \& Cullen, P. J. (2013). A global analysis of SNX27-retromer assembly and cargo specificity reveals a function in glucose and metal ion transport. Nature cell biology, 15(5), 461.

Strochlic, T. I., Schmiedekamp, B. C., Lee, J., Katzmann, D. J., \& Burd, C. G. (2008). Opposing activities of the Snx3-retromer complex and ESCRT proteins mediate regulated cargo sorting at a common endosome. Molecular biology of the cell, 19(11), 4694-4706.

Vilariño-Güell, C., Wider, C., Ross, O. A., Dachsel, J. C., Kachergus, J. M., Lincoln, S. J., ... \& Behrouz, B. (2011). VPS35 mutations in Parkinson disease. The American Journal of Human Genetics, 89(1), 162-167.

Wang, H., Lu, B., Castillo, J., Zhang, Y., Yang, Z., Lindeman, A., ... \& Cong, F. (2016). Tankyrase inhibitor sensitizes lung cancer cells to EGFR inhibition via stabilizing 
angiomotins and inhibiting YAP signaling. Journal of Biological Chemistry, 291(29), 15256-15266.

Wang, J. Y., Zhuang, Q. Q., Zhu, L. B., Zhu, H., Li, T., Li, R., ... \& Zhu, J. H. (2016). Meta-analysis of brain iron levels of Parkinson's disease patients determined by postmortem and MRI measurements. Scientific Reports, 6.

Wang, T., Wei, J. J., Sabatini, D. M., \& Lander, E. S. (2014). Genetic screens in human cells using the CRISPR-Cas9 system. Science, 343(6166), 80-84.

Warde-Farley, D., Donaldson, S. L., Comes, O., Zuberi, K., Badrawi, R., Chao, P., ... \& Maitland, A. (2010). The GeneMANIA prediction server: biological network integration for gene prioritization and predicting gene function. Nucleic acids research, 38(suppl_2), W214-W220.

Zavodszky, E., Seaman, M. N., Moreau, K., Jimenez-Sanchez, M., Breusegem, S. Y., Harbour, M. E., \& Rubinsztein, D. C. (2014). Mutation in VPS35 associated with Parkinson's disease impairs WASH complex association and inhibits autophagy. Nature communications, 5 .

Zimprich, A., Benet-Pagès, A., Struhal, W., Graf, E., Eck, S. H., Offman, M. N., ... \& Rossle, S. C. (2011). A mutation in VPS35, encoding a subunit of the retromer complex, causes late-onset Parkinson disease. The American Journal of Human Genetics, 89(1), 168-175. 